\title{
A decade of innovation and progress in understanding the morphology and structure of heterogeneous polymers into rigid confinement
}

\author{
Salim $\mathrm{Ok}^{1, *}, \$$, Marylène Vayer ${ }^{2, \$}$, Christophe Sinturel $^{2, \$}$ \\ ${ }^{1}$ Petroleum Research Center, Kuwait Institute for Scientific Research, P.O. Box 24885 Safat, 13109, Kuwait \\ ${ }^{2}$ Interfaces, Confinement, Matériaux et Nanostructures (ICMN), CNRS-Université d’Orléans, UMR 7374, \\ 1b, Rue de la Férollerie, C.S. 40059, 45071 Orléans Cedex 2, France \\ ${ }^{*}$ Correspondence, E-mail: sok@uos.de \\ \$Equal Contributors \\ When confined into nanoscale domains, polymers generally encounter changes of their structural, thermodynamics \\ and dynamics properties compared to those in bulk, due to the high amount of polymer/walls interfaces and limited \\ amount of matter. The present review specifically deals with the confinement of heterogeneous polymers (i.e. polymers \\ blends and block copolymers) into rigid nanoscale domains (i.e. bearing non-deformable solid walls) where the \\ processes of phase separation and self-assembly can be deeply affected. This review focuses on the innovative \\ contributions of the last decade (2010-2020), giving a summary on the new insights and understanding gained in this \\ period. We conclude this review by giving our view on the most thriving directions for this topic.
}




\section{List of Abbreviations}

AAO: Anodic Aluminum Oxide. n: Avrami index. BCP: Block Copolymer. DPD: Dissipative Particle Dynamics. DS: dielectric spectroscopy. DSC: Differential Scanning Calorimetry. DDFT: Dynamic Density Functional Theory. FTIR: Fourier Transform Infrared. IMDS: Intermaterial Dividing Surface. iPS: isotactic poly(styrene). LMCM: Long MultiCompartment Micelles. $\mathrm{M}_{\mathrm{w}}$ : molecular weight. MD: Microdomain. NMP: N-Methyl-2-pyrrolidone. nSVA: non-Solvent Vapor Annealing. NMR: Nuclear Magnetic Resonance. MD: Molecular Dynamics. MC: Monte Carlo. MCM: MultiCompartment Micelles. 1D: One-Dimensional. POM: Polarizing Optical Microscopy. PBD: Poly(butadiene). PCL: Poly( $\varepsilon$-caprolactone). PDMS: Polydimethylsiloxane. PPO: Poly (2,6-dimethylphenylene oxide). P3DDT-b-PEMA: Poly(3-dodecylthiophene)block-poly(ethyl methacrylate). PE: Poly(ethylene). PEO: Poly(ethylene oxide). PE-b-PS: Poly(ethylene)-blockpoly(styrene). P3HT: Poly(3-hexylthiophene). PLLA: Poly(L-lactic acid). PMMA: Poly(methyl methacrylic acid). PP: Poly(propylene). PS-b-P4VP: Poly(styrene)-block-poly(4-vinylpyridine). PS: Poly(styrene). PS- $b$-PBD: poly(styrene)block-poly(butadiene). PS-b-PDMS: Poly(styrene-block-dimethylsiloxane). PS-b-PI: poly(styrene)-block-poly(isoprene). PI-b-PS-b-P2VP: Polystyrene-block-polyisoprene-block-poly(2-vinylpyridine). PS-b-PLLA: Poly(styrene)-block-poly(Llactic acid). PS-b-PMMA: Poly(styrene)-block-poly(methyl methacrylate). PS-b-PVP: Poly(styrene-block-vinyl pyridine).

PTFE: Poly(tetrafluoroethylene). PVA: Poly(vinyl alcohol). P(VDF-TrFE): Poly(vinylidene-trifluoroethylene). SEM: Scanning Electron Microscopy. SCF: Self-Consistent Field. SAXS: Small-Angle X-ray Scattering. SMA: Small Spherical Assembly. SVA: Solvent Vapor Annealing. 3D: Three-Dimensional. 2D: Two-Dimensional. TEM: Transmission Electron Microscopy. WAXS: Wide-Angle X-ray Diffraction. XRD: X-ray Diffraction. XPS: X-ray Photoelectron Spectroscopy. 
Table of Contents

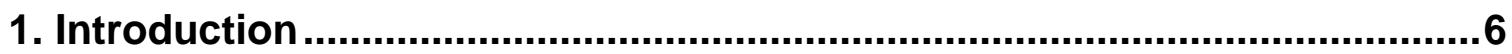

2. Hard templates and polymer infiltration processes...................................8

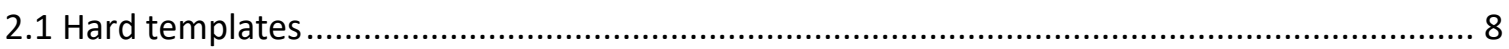

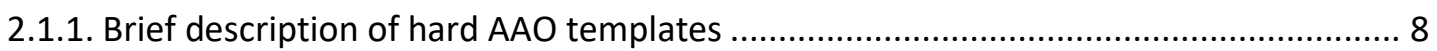

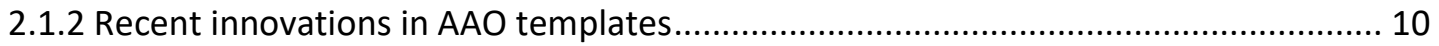

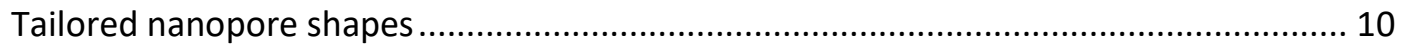

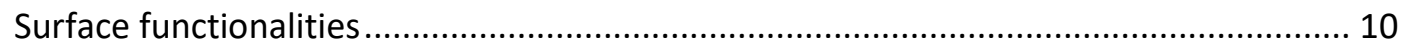

Combination of tailored sizes and surface functionalities ........................................... 11

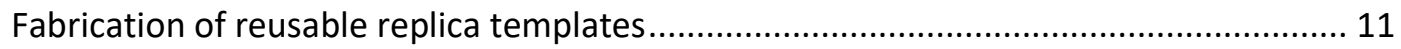

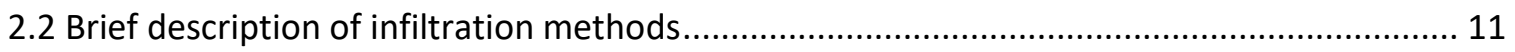

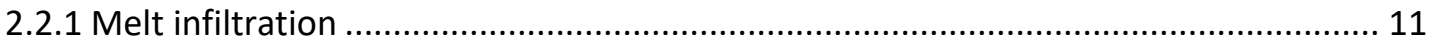

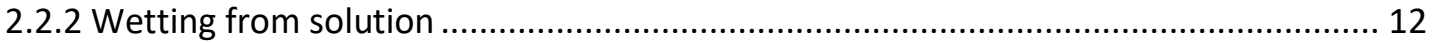

2.2.3 Other new methods or innovations in existing methods ......................................... 13

Solvent annealing induced nanowetting in templates (SAINT) ................................... 13

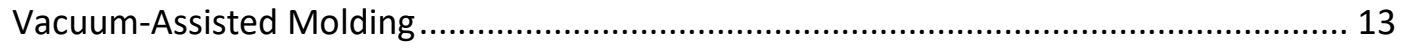

Microwave annealing induced nanowetting (MAIN) ................................................ 13

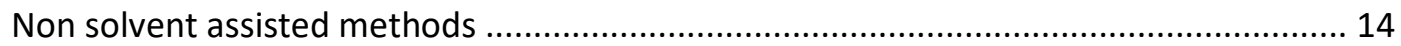

Combination of thermal imprint and AAO templates ................................................. 15

3. Polymer blends under confinement ........................................................15

3.1. Phase separation in the bulk and parameters influencing the stability of polymer blends .. 15

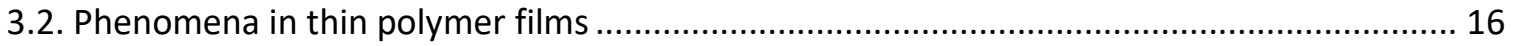

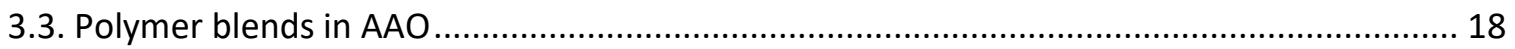

3.3.1 Morphology induced by surface affinity with the pore walls .................................. 19

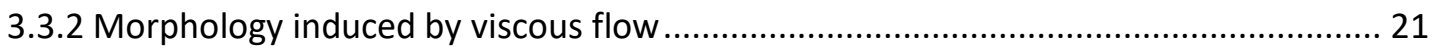

3.3.3 Morphology induced by sequential introduction.................................................... 23

4. Block copolymers under confinement .......................................................25

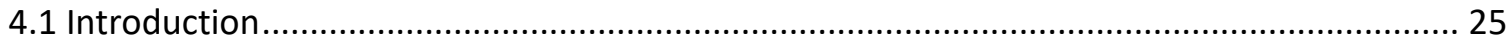

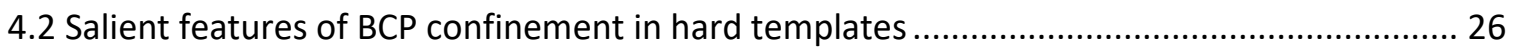

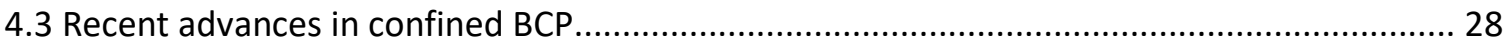




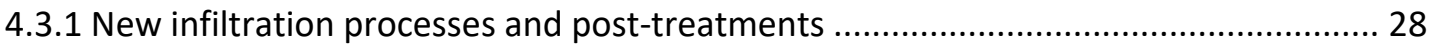

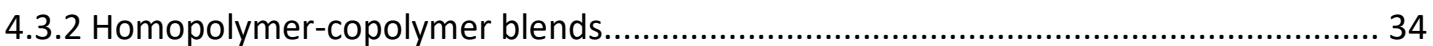

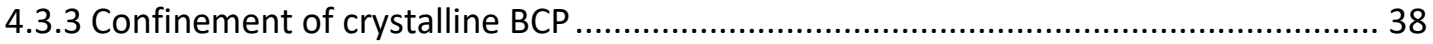

5. Dynamics of confined polymers................................................................44

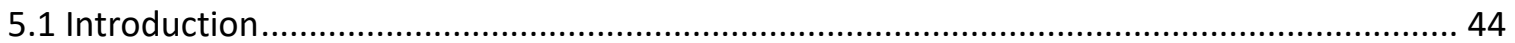

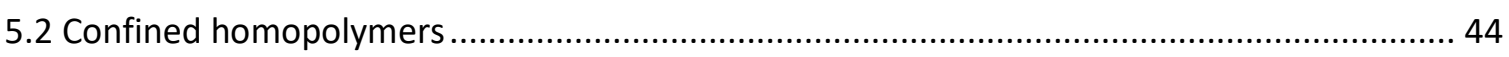

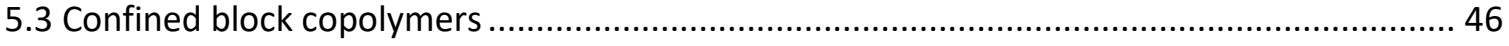

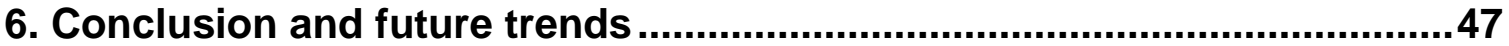

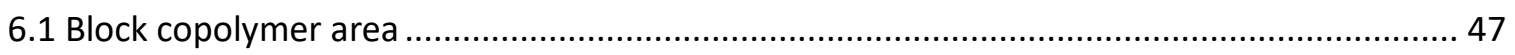

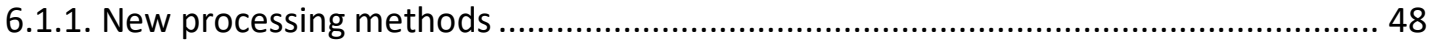

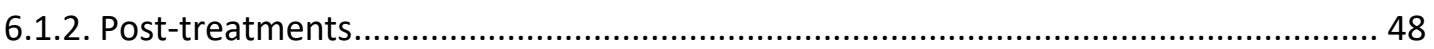

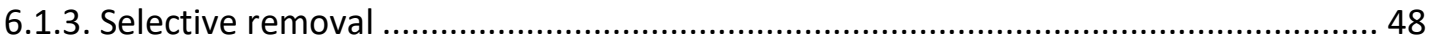

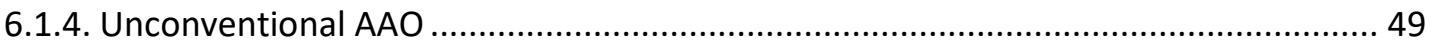

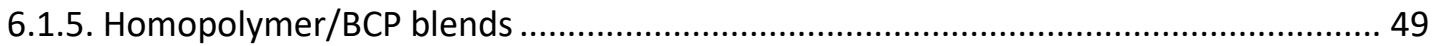

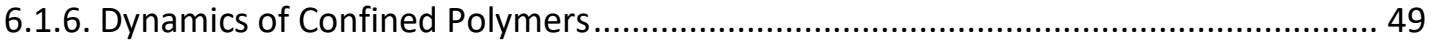

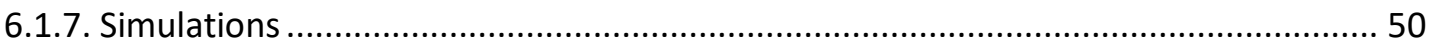

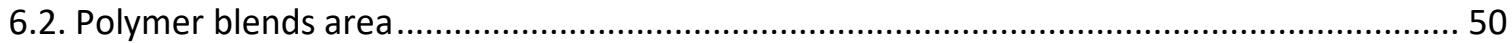

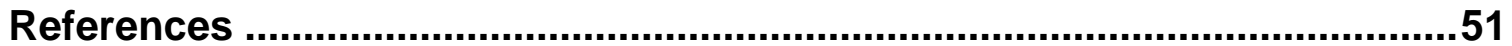


Dr. Salim Ok has a Ph.D. in

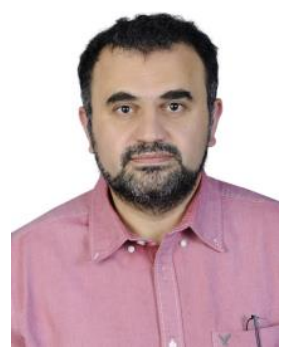

NMR spectroscopy analysis

of fluoropolymers in solid-

state, in the melt at high

temperatures, and at high-

pressures in solution in

supercritical carbon dioxide from Technical University

of Dresden. Dr. Ok has been working with geologically

important confined fluids. He has been researching

polymers under nano-confinement, polymer thin

films, characterization of fluoropolymers, and surface

modifications. He has contributions to the analysis of natural products such as olive oil and focuses on connecting natural product chemistry to materials science by using natural chemicals for preparing nanomaterials.

Dr. Marylène Vayer is a researcher at the CNRS (French center of scientific research). She holds an engineering degree in chemistry from the École Nationale Supérieure de Chimie de Strasbourg, France (1983). She received her Ph.D. in materials science from the University of Nancy, France (1987). She was appointed as a CNRS researcher in 1988. She is currently working at the laboratory ICMN

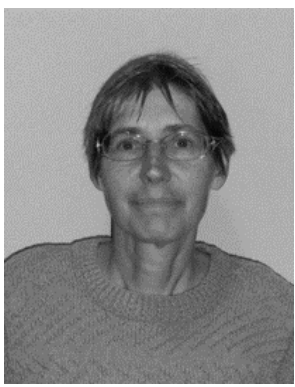

(Interface, Confinement,

Materials, and

Nanostructuration) in

Orleans (France). Her current

research interest is the nano

structuration of polymers in thin films.

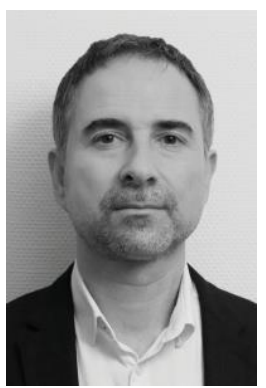

Dr. Christophe Sinturel received his Ph.D. in Polymer Science in 1998 from the University of Clermont-Ferrand (France). He spent one year at the University of Brighton (UK) in 1999 as Postdoctoral Research Associate before being appointed as an associate professor the same year at the University of Orléans (France). In 2010 he got a full-professor position and he is now conducting his research at ICMN, a joint Research Institute of the CNRS and the University of Orléans. His current research interests concern mainly polymer in thin films such as polymer blends, nanostructured polymers, polymer nanocomposites, and block polymers. 


\section{Introduction}

The infiltration of polymers into nanoporous templates (i.e. porous materials) is of significant interest in both fundamental science and technological applications. Depending on the process, various nanostructures such as hollow nanotubes, nanorods, nanospheres, nanodiscs, etc. can be formed. Such nanostructures have received a great deal of attention because of their potential applications in drug delivery, sensors, unconventional organic electronics, and conducting materials, to name but a few. $\cdot^{1-2}$ From a fundamental point of view, the confinement imposed by the spatial restriction can influence the characteristics of the confined polymer including its thermodynamics, dynamics, conductivity, phase properties and crystallization behavior.

In the present review, among other issues, we are interested in the changes in the phase properties (morphology and nanostructure geometry) and crystallization behavior of heterogeneous polymers such as polymer-polymer blends or block copolymers (BCPs) under two-dimensional (2D) confinement. Note that generally, the confinement of polymers and/or BCPs can be achieved in various geometries ranging from one (thin films or ultra-thin films), two (cylindrical environment in inorganic nanoporous templates such as nanoporous alumina or cylinders in block copolymers) to three (droplets of polymers or spheres in $\mathrm{BCP}$ ) dimensions. Among different templates, anodic aluminum oxide (AAO) templates are the most widely used to study hard 2D confinement due to the facility of their preparation and the ease of polymer infiltration. In 2D confinement in AAO templates, the properties of the polymers are influenced by the type of nanostructures formed (nanorods, nanotubes, and/or nanospheres), the degree of the confinement (pore diameter), and the surface properties of the nanoporous templates $(-\mathrm{OH}$ decorated pore walls coming from AAO template production versus functionalized surfaces).

Following the pioneering work by Martin and co-workers in the $90 \mathrm{~s}^{3-6}$, confinement in porous materials received plenty of interest in the 2000s, thanks to advances in the control of AAO membrane formation. To name but a few, the studies by Steinhart in $2002^{7}$ and Song in $2004^{8}$ showed the formation of polymer nanotubes or nanorods by filling AAO membranes with polymer melt and/or polymer dissolved in a solvent. Following these early efforts, towards the end of the $2000-2010$ decade, Steinhart ${ }^{9}$ covered in his review the various ways in which nanoporous AAO templates could be used for the production of mesoscopic fine structures such as polymeric nanofibers and the realization of previously unknown confinement-induced morphologies with novel and outstanding characteristics. Particularly, relationships between optical, electronic, and ferroelectric properties and their characteristics such 
as morphology, crystal size, and orientation were a major focus. Therefore, finding proper approaches was essential to govern the establishment of the internal morphologies of the confined polymers to develop novel polymeric nanomaterials with enhanced capabilities. Towards the end of the first decade, two methods of wetting were established: i) melt wetting, and ii) wetting from polymer solutions. These methods were applied mainly to homopolymers. The number of experimental studies on confined block copolymers in hard templates was still overwhelmed ${ }^{10-14}$ by the large number of simulation studies. ${ }^{15-16}$ The infiltration of homopolymer blends was even less documented.

In the last decade (2010-2020), the research effort was pursued, generating additional literature, summarized in detailed reviews $s^{15-20}$, including some very recent ones. ${ }^{21-26}$ Table S1 in the Supporting Information summarizes the main topics covered by these reviews and shows that, despite a sizeable number of contributions, the question of structure and morphology in AAO was seldom addressed in a combined way for either polymer blends or copolymers. Four recent reviews summarize the crystallization of polymers upon confinement in AAO hard templates ${ }^{17,} 20,23,27$ as well as soft confinement crystallization. ${ }^{23,} 28 \mathrm{Wu}$ et al. ${ }^{21}$ also discuss the various polymer nanostructures generated by confining the polymers in AAO templates using homopolymers, polymer blends, or BCPs. The effects of confinement on the polymer structures, the dynamics, the mechanical properties, and the rheological properties have been reviewed by various reports. ${ }^{18,} 19,25$ Examples of confined polymer applications have been reviewed by ${ }^{29}$ in nanobiotechnology, and for stimuli response applications. ${ }^{26}$

On the specific question of the morphology and structure of heterogeneous polymers, the end of the last decade calls for a new review article summarizing what type of questions on confined polymers have been answered and which ones remain to be addressed in the coming decade. The key differentiator of the present review is that it combines experimental contributions of the last decade for both polymer blends and BCPs confined in hard nanoporous AAO templates (with a particular focus on crystalline BCPs), offering an up-to-date digest of innovative ideas in generating novel nanostructures out of polymers in a confined environment. Although simulation studies are a source of inspiration for potential novel experiments, it is beyond the scope of this paper to review the considerable amount of simulation work available.

The contributions on BCPs in confined geometry increase rapidly, and a great number of scientific and technical articles have been published in this area from the perspective of chemistry, materials science, and physics. The increase of the literature 
works on confined polymers makes it difficult to include all the recent studies. Therefore, we mainly focus on the experimental studies of the confined BCPs, blends of homopolymer/homopolymer and $\mathrm{BCP} /$ homopolymer as important examples of heterogeneous polymers. Besides, we also attempt to cover mainly the literature of the last decade. As a result, in advance we apologize if we miss referencing a little portion of the relevant and important literature.

The current review is divided into three main sections. In the first section, we introduce the AAO templates and processes of polymer infiltration. This section includes a description of the production and properties of AAO hard templates, some recent innovations in AAO templates (such as the preparation of tailored pores, different patterns in nanoporous AAO, and surface functionalization), and a description of infiltration methods (including some very recent developments). In the second section, we detail the case of the confinement of homopolymer blends. After introducing the basic concepts on polymer blends in thin films, the behaviors of polymer blends inside AAO templates are described, with a strong emphasis on both the major driving force for infiltrating polymer blends into the nanopores of the AAO templates and the final properties of polymer blends in confined geometry. In the third section, the characteristics of BCPs confined in nanoporous AAO templates are discussed with a focus on how the morphology (self- assembly) and the crystallization behavior of BCPS deviate in confined geometry from their bulk behavior.

The review concludes with the lessons learned in the previous decade and with a short discussion on potential new research avenues in the next few years.

\section{Hard templates and polymer infiltration processes 2.1 Hard templates}

\subsubsection{Brief description of hard}

\section{AAO templates}

Among several materials that can be used to confine polymers, hard AAO templates have been the focus of the majority of polymer confinement studies due to the facile infiltration of polymers into their ordered porous network. In $19^{\text {th }}$ century, Buff recognized the possibility of oxidizing aluminum electrochemically in an aqueous solution to generate an oxide layer thicker than the ones formed at ambient atmosphere. $^{30}$ This concept was defined as "anodization" since the aluminum whose surface is to be modified constitutes the anode in an electrolytic unit cell. ${ }^{31}$ In 1995, Masuda and Fukuda established an inexpensive experimental method allowing to generate a periodic porous network over a relatively large surface by tuning parameters including applied voltage, electrolyte selection, and temperature. ${ }^{32}$ Hence, it becomes possible to prepare nanoporous templates with pre-designed porous features such as pore diameter and length, porosity, and interpore distance, etc for specific applications. ${ }^{33}$ This high level 
of flexibility in the nanoporous structure down at the nanometer scale provides advantages in fine-control of different materials including polymers.

The experimental process of AAO template fabrication contains the two-step anodization of aluminum. Note that as of today anodization is a facile and low-cost electrochemical oxidation procedure of metal surface oxidation over large areas..$^{33}$ Anodization of aluminum in aqueous solutions result in anodic oxide surfaces with two differing structural features: i) the barrier-type nonporous oxide layer, and ii) the porous-type oxide layers, where the two types of anodic-oxides differ mainly in kinetics of the oxide

templates, at the end of the first anodization, an irregular AAO layer is formed. Upon removing the first layer, the second anodization is completed. The remains of the first anodization act as a mask for the fabrication of the new ordered features in hexagonal arrays of parallel cylindrical nanopores. Fig. $1 \mathrm{a}$ and $1 \mathrm{~b}$ display typical side-view and top-view SEM images of AAO templates. ${ }^{34-35}$

The tunability of pore diameter and ease of surface modification of nanopore walls make AAO ideal templates for studying different polymers in confined geometry. ${ }^{17,}{ }^{21}$ Currently, AAO templates are by far the most commonly utilized nanoporous templates for the production of one- and twolayer growth. ${ }^{31}$ In generating nanoporous AAO
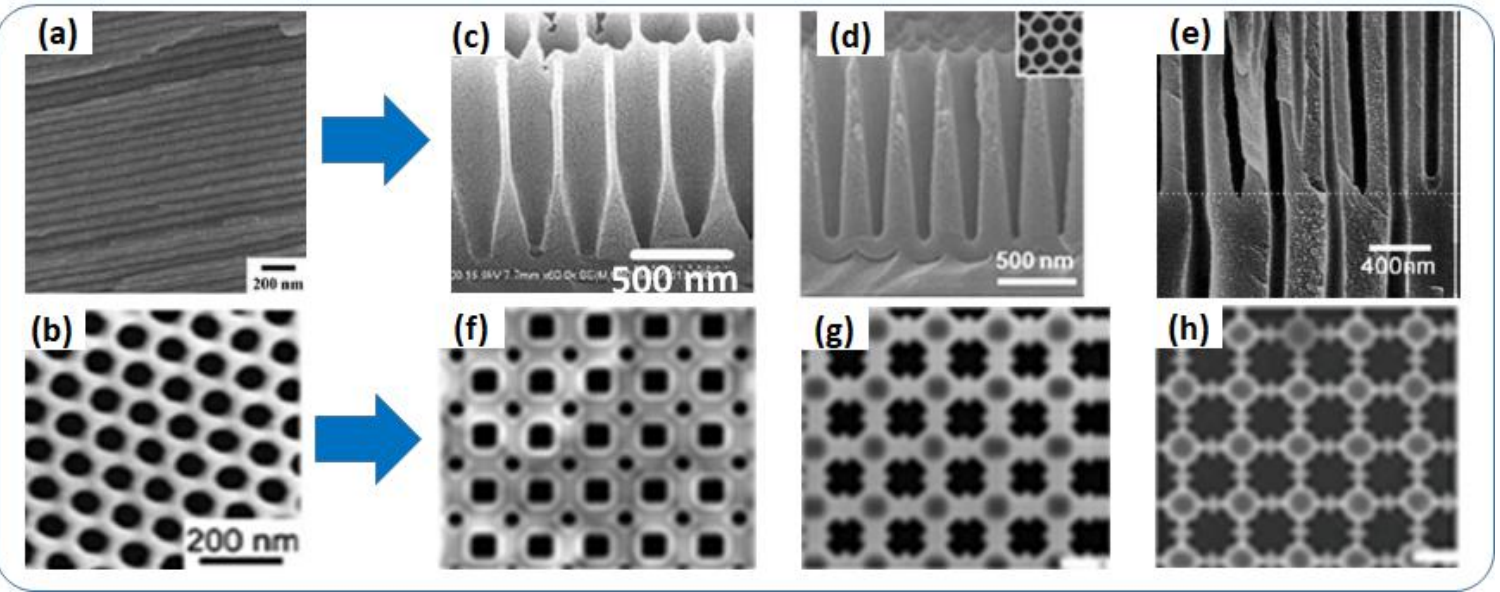

Fig. 1. Progress in hard AAO template design: examples of SEM images. Top: from cylindrical shape (a) Reproduced from Polymer 110, 27334, Copyright 2017, with permission from Elsevier, to tailored shapes (c) Reproduced from ACS Appl. Mater. Interfaces 4, 5678 ${ }^{41}$, Copyright 2012, with permission from the American Chemical Society, (d) Reproduced from Macromolecules 52, 480342, Copyright 2019, with permission from the American Chemical Society, (e) Reproduced from J. Solid State Electrochem. 17, 1931 ${ }^{45}$, Copyright 2013, with permission from Springer. Bottom: from hexagonal array (b) Reproduced from Soft Matter 7, 187335, Copyright 2011, with permission from Royal Society of Chemistry, of nanopores to more complex arrays (f), (g), (h) Reproduced from Nature Nanotech. 12 (2017) 24446, Copyright 2017, with permission from Nature Publishing Group. 
dimensional nanomaterials. ${ }^{36}$ AAO templates have several advantages: i) the ease of tuning the nanopore aspect ratios (length/diameter) by adapting the preparation conditions of the composition of the electrolyte, anodization time, voltage, and current level, ii) low-cost production, iii) homogeneity of nanopore arrangement, and iv) stability at high temperatures. ${ }^{18}$ The pore diameter of AAO templates can range typically from 20 to $400 \mathrm{~nm}$ with possible lengths up to $200 \mu \mathrm{m} .{ }^{29,} 37,38$ Varying the anodization conditions result in various morphologies of the porous structure in AAO templates leading to the formation of innovative features at the nanometer scales. $^{33}$

\subsubsection{Recent innovations in AAO} templates

To go beyond the simple honeycomb structure with cylindrical pores having a monodisperse pore size distribution, AAO template fabrication has been improved, resulting in tailored pore shapes and sizes, assembled in different patterns and with different surface functionalities. Jani et al. ${ }^{39}$ and Rath and Theato $^{26}$ reported on the advances of AAO template engineering and their applications.

\section{Tailored nanopore shapes}

Li et al. ${ }^{40-41}$ proposed a multi-step process (one step of hard anodization-etching peeling and multi-steps of mild anodization-etching widening) to create AAO nanoporous templates with tailored pore shapes such as linear, whorl-embedded cones, funnels, pencils (Fig. 1(c)), parabolas, and trumpets. Shapes such as cones, truncated cones, and cylinders were developed by Kim et al. ${ }^{42}$ (Fig. 1(d)), while Raoufi and Schönherr fabricated AAO templates with modulated pore diameters ${ }^{43}$ (Fig. S1).

Non-honeycomb patterns

Multi-tiered branched nanoporous AAO templates with an array of pores branching into smaller pores in successive tiers like roots were developed by $\mathrm{Ho}$ et al. ${ }^{44}$ Other complex patterns showing tree-like structures (Fig. $1(\mathrm{e}))^{45}$ or multisets of pores with different geometries and patterns (Fig. 1(fh) $)^{46}$ have been fabricated. In the latter case, a multistep method is applied with a first imprinting step to prepare pores with different geometries: square, circle, and four-cross dots arranged in different types of patterns (Fig. S2).

\section{Surface functionalities}

Modification of the pristine hydroxyl (-OH) decorated pore walls of AAO templates is possible via reaction with, for example, trichloro $(1 \mathrm{H}, 1 \mathrm{H}, 2 \mathrm{H}, 2 \mathrm{H}$ perfluorooctyl)silane ${ }^{47}$, octadecyl phosphonic acid ${ }^{48}$, and polystyrene (PS). ${ }^{42}$ These surface modifications of the nanopore walls make it possible to tune the melting attitude, crystallization, affinity, and localization of the confined polymers. 
Combination of tailored sizes and surface functionalities

Liu et al. ${ }^{49}$ generated AAO templates with pores exhibiting simultaneously different sizes and surface properties. To prepare pores with two different sizes, a process was developed with a twostep anodization followed by pore widening and a third anodization. To prepare pores with two surface properties, a two-step anodization method was followed by surface functionalization and a third anodization (Fig. S3). These procedures were combined to fabricate pores with tailored sizes and surface properties.

\section{Fabrication of reusable replica templates}

A major advance concerns the fabrication of reusable replica templates using AAO membranes developed by Zhang et al. ${ }^{50}$ using nanoimprint lithography methods. The method involves the fabrication of a polyurethane acrylate mold using an AAO template to produce UV resin replicas enabling confinement of the polymer in the nanopore. This has been used to generate an anti-reflective porous structure (Fig. S4). However, to date this method has not been used to produce confined polymer structures such as nanorods or nanotubes.

\subsection{Brief description of infiltration methods}

\subsubsection{Melt infiltration}

The infiltration of polymers into nanoporous AAO templates is possible in the molten state of polymers, either above the glass transition $\left(T_{g}\right)$ for amorphous polymers or above the melting temperature $\left(T_{m}\right)$ for semi-crystalline polymers. ${ }^{18}$ One of the pioneering studies by Steinhart et al. showed the formation of polystyrene (PS) and poly (tetrafluoroethylene) (PTFE) nanotubes with monodisperse size distribution and uniform orientation by wetting AAO templates via melt infiltration. ${ }^{7}$ Two infiltration regimes were established: i) precursor wetting corresponding to a complete wetting regime, ii) capillary wetting with a partial wetting regime.

In precursor wetting, the driving force for filling the AAO nanopores with high surface energy for the polymers with lower surface energy is that the free energy of the system is easily lowered.7, 21 In this complete wetting case, the precursor film melts and spreads on the inner walls of the AAO nanopores. ${ }^{51}$ When the nanopore diameter is two-fold greater than the thickness of the precursor film, hollow nanotubes are generated upon quenching. If the pore diameter of AAO is lowered to twice the gyration radius $\left(R_{g}\right)$ of confined polymers, solid rods are observed. ${ }^{52}$

Capillary wetting is observed if polymer melts are confined in the AAO template where the establishment of a precursor film is suppressed. In such a partial wetting regime, nanopores are considered as nanosized capillaries, and polymer melts gradually fill the nanopores by capillary pressure. If the 
polymer inside the nanoporous template is cooled down to a temperature lower than either $T_{g}$ or $T_{m}$, the polymer melts solidify, yielding nanorods. The existence of a meniscus at the tip of the generated nanorods is a mark of capillary action. ${ }^{21}$

In the case of homopolymer melts, a change from complete wetting to capillary wetting occurs, related to an increase in the viscosity of the polymer melt. PS with an average molecular weight $\left(\mathrm{M}_{\mathrm{w}}\right)$ of 30 $\mathrm{kg} . \mathrm{mol}^{-1}$ yielded solid rods in AAO membranes with a pore diameter of $200 \mathrm{~nm}$ upon annealing for $2 \mathrm{~h}$ at 130 ${ }^{\circ} \mathrm{C}$. For an infiltration temperature of $205{ }^{\circ} \mathrm{C}$, PS nanotubes were instantaneously obtained. ${ }^{53}$ Nanotubes are preferred for low $M_{w}$ polymers, while nanorods are mainly seen with high $\mathrm{M}_{\mathrm{w}}$ polymers. Both precursor and capillary wetting approaches can result in nanorods but with different kinetics. ${ }^{9}$ Filling nanoporous hard templates by microphase-separated BCPs mostly occurs via capillary wetting. ${ }^{54}$ Practically, melt infiltration can be achieved by putting bulk polymer or polymer film in contact with AAO membranes at sufficient temperature.

\subsubsection{Wetting from solution}

Wetting the nanopores with polymers from solution ${ }^{21}$ can be achieved either by placing the polymer solution on top of the template ${ }^{7}$, or by spin coating the polymer solution on the template ${ }^{55}$, or by immersing the template in the polymer solution. ${ }^{56}$ The polymer solutions wet the nanopores by capillary force. As in melt wetting, solution wetting also results in morphologies of nanotubes and nanorods depending on the wetting regime, so characterized by the spreading coefficient. ${ }^{51}$ In the partial wetting regime, the polymer solutions result in polymer nanorods, while in the full wetting regime, polymer solutions establish wetting layers. ${ }^{21}$ The final morphology of the confined polymer will be affected by the solvent evaporation, which is hard to control. Various polymer nanostructures can be obtained by varying experimental parameters in the solution method including polymer type, the $M_{w}$ of the polymer, solvent type, or concentration of the solution. ${ }^{57}$ and references therein Using dilute polymer solutions will result in nanotubes, while concentrated polymer solutions will yield solid nanorods. ${ }^{58}$ Each infiltration method has its advantages and disadvantages. The advantage of the solution method is the preparation of versatile polymer nanostructures, while the disadvantage is that the experimental parameters are difficult to control, which might be the reason for the non-reproducible results. ${ }^{57}$ Upon evaporation of the solvent, residual polymer molecules on either side of the template may cause problems in characterizing the probes. Incomplete solvent evaporation and lack of enough material inside the nanopores are other disadvantages of wetting with the solution method. ${ }^{59}$ 
In melt infiltration, the infiltration temperature can be freely selected, depending on the $T_{g}$ or the $T_{m}$ of the polymer to be confined. However, the long infiltration times of several days or sometimes even weeks, and potential thermal degradation are major disadvantages of the melt infiltration approach. In melt infiltration, substantially faster infiltration of a polymer in the complete wetting regime compared to the capillarity method (partial wetting regime) is an advantage..$^{59}$

\subsubsection{Other new methods or} innovations in existing methods

Solvent annealing induced nanowetting in templates (SAINT)

Another method of polymer infiltration in AAO hard templates is solvent annealing induced nanowetting in templates (SAINT) that has also been proposed for confining polymers such as polystyrene (PS) and poly(methyl methacrylate) (PMMA) in AAO nanopores. ${ }^{47,}$, $60-64$ In this method, a polymer film is deposited on a substrate, and an AAO membrane is deposited on top of the polymer film. This system is placed in a closed and sealed container in the presence of solvent vapor that plasticizes the polymer, which is thus able to flow into the AAO nanopores. This method has been used to confine thermosensitive polymers such as poly(vinyl chloride) (PVC) or conducting polymers, for example, poly(3-hexylthiophene) (P3HT). ${ }^{64}$ The SAINT method could overcome the thermal degradation problem encountered in melt infiltration. ${ }^{61}$

\section{Vacuum-Assisted Molding}

In vacuum-assisted infiltration (Fig. S5) ${ }^{65}$, external forces are the main driving forces for polymer infiltration, a major difference compared to that of wetting-based techniques. The vacuum-assisted approach mainly depends on viscosity. This approach is the method of choice for polymers, in particular when the polymers cannot spontaneously infiltrate into the nanopores. ${ }^{59}$ In their pioneer work, Cepak and Martin ${ }^{66}$ prepared polymeric nanotubes by vacuum "filtration" of a polymeric solution in the AAO membranes. This technique can generate nanowires with a high aspect ratio using cross-linking polymers such as polydimethylsiloxane (PDMS) and thermoplastics such as PTFE or polyamide. In vacuumassisted molding, both polymer melts and thermoset elastomers can be confined in nanoporous AAO templates.

Microwave annealing induced nanowetting (MAIN)

The MAIN method, using microwave rather than thermal annealing, can be considered as a derivative of melt wetting. ${ }^{21}$ Chang et al. ${ }^{57}$ developed the MAIN technique to generate one-dimensional (1D) polymer nanomaterials out of PS using AAO templates. As soon as microwave annealing is performed, the polymer chains are infiltrated into the AAO templates, 
and it is possible to control the morphologies of the polymer nanomaterials by governing the annealing conditions and parameters. The growth rates of the polymer nanomaterials generated by the MAIN approach are higher than those fabricated by the traditional thermal annealing technique. The MAIN method provides both a time-saving approach for governing the morphologies of confined polymer nanomaterials and a scientific case to study the influence of microwave annealing on the confined polymeric materials. ${ }^{57}$ Chang et al. ${ }^{67}$ used the MAIN approach to infiltrate poly(styrene-blockdimethylsiloxane) (PS-b-PDMS) into AAO nanopores. For this purpose, the AAO is placed above a thin film of the $\mathrm{BCP}$ and then put in the microwave annealing device to anneal at $190^{\circ} \mathrm{C}$ for $15 \mathrm{~min} .{ }^{67}$ Note that this technique has already been used by several authors to achieve ordered morphologies in block copolymer thin films. ${ }^{68-69}$

$$
\begin{aligned}
& \text { Non solvent assisted methods } \\
& \text { Another important approach recently }
\end{aligned}
$$
two steps: the first one is a classical wetting from polymer solution and the second one is a wetting with a non-solvent of polymers. Note that for polymer thin films, it was shown that a mixture of miscible solvent and non-solvent could change the morphology of the polymer thin films. ${ }^{70}$ In the second step of wetting with a non-solvent of polymers (e.g. water), a stronger interaction between the non-solvent ${ }^{71-72}$ and the alumina surface causes the polymer solution to be isolated in the center of the nanopores, resulting in polymer nanorods ${ }^{71}$ or nanospheres. ${ }^{72}$ Thermal annealing of these nanostructures in the AAO pores resulted in the formation of curved nanodiscs. ${ }^{72}$

The use of a non-solvent in an annealing post-treatment was also proposed by Huang et al. ${ }^{73}$ and resulted in polymer nanorods and nanospheres. In this case, nanofibers obtained by wetting AAO from dimethyl formamide (DMF) solution and removed from the templates are further annealed in ethylene glycol, resulting in nanospheres. The size of these nanostructures can be tuned by the annealing temperature and time. 


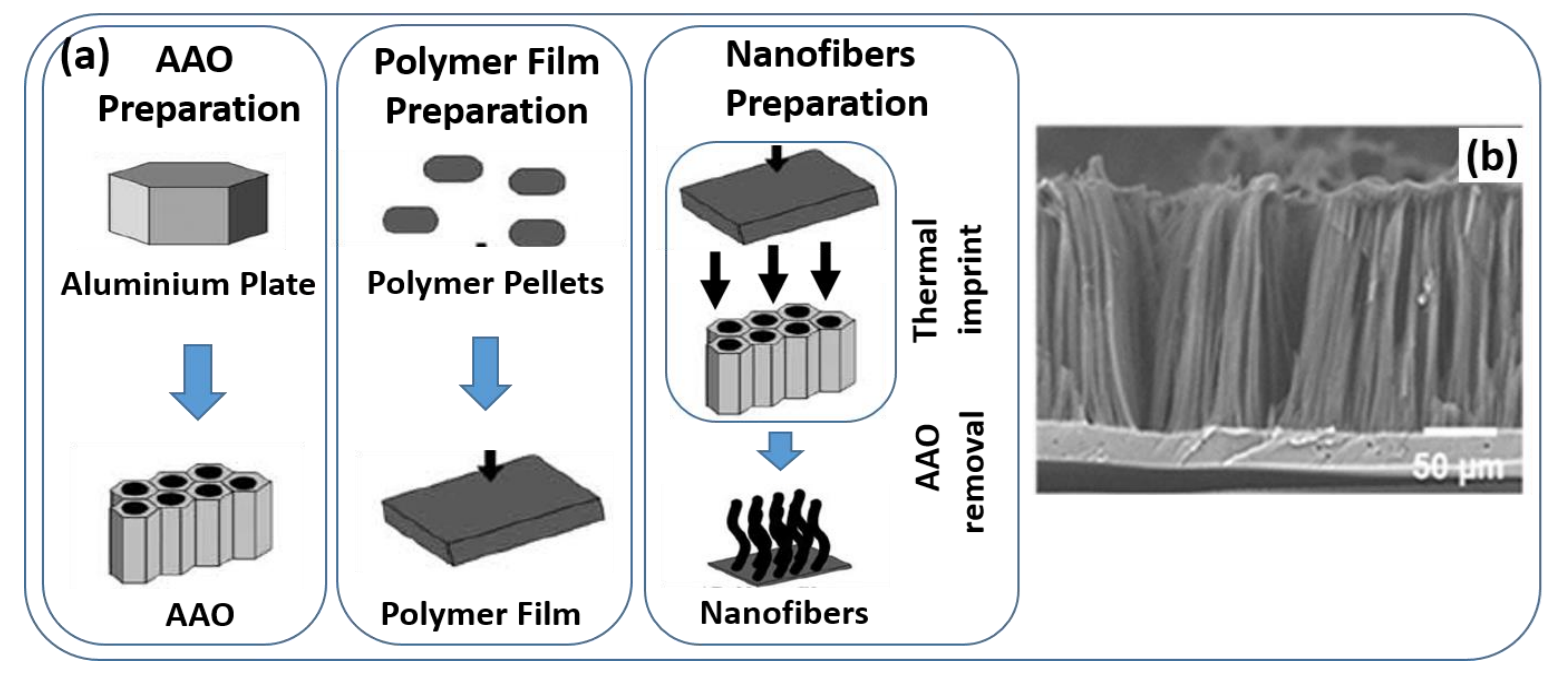

Fig. 2 Fabrication of polymer nanofibers using the thermal nanoimprint method: schematic of the process (a); SEM image of PP nanofiber array (b). Reproduced from Polym. Eng. Sci. 57, 21474, Copyright 2017, with permission from Wiley.

Combination of thermal imprint and $A A O$ templates

Among the latest methods developed to confine polymers in AAO nanoporous templates, Muanchan et al. ${ }^{74}$ proposed to use the combination of a thermal nanoimprint method and AAO templates and studied the influence of the processing parameters such as temperature, pressure, and time on the length of polymer fibers (Fig. 2). PS and poly(propylene) (PP) were used. With a $50 \mathrm{~nm}$ pore diameter, fibers with an aspect ratio higher than 1000 were prepared. This method leads to an increase in the polymer chain packing with an increase in $T_{g}$ for PS fibers, a decrease in crystallinity for PP, and a reduction in mechanical properties.

\section{Polymer blends under confinement}

Before examining the literature specifically dealing with the phase behavior of polymer blends in AAO (section 3.3), we will first recall the main phenomena and parameters implicated in bulk and thin films (sections 3.1 and 3.2 , respectively). The purpose of these two preliminary sections is not to propose a full review of polymer blends in bulk and thin films but rather to present the salient features of the phase behavior in such an environment since the confinement of polymer blends in AAO cylindrical porosity proceeds via phenomena derived from those arising in bulk and thin films.

\subsection{Phase separation in the bulk and}

parameters influencing the stability of polymer blends

Most polymers are incompatible, leading to a phase separation when mixing counterparts with 
different chemistries. The thermodynamics of polymer blends in bulk is described with the well-known FloryHuggins theory. ${ }^{75-76}$ The change of free energy in a binary blend results from a contribution of the enthalpy of mixing which depends on the incompatibility between the segments of polymers ${ }^{77}$, and a contribution of entropy, which always decreases the free energy of mixing. Beyond thermodynamics, blend processing is of prime importance. The thermodynamically stable final morphology is not always reached, leaving the blend in metastable or intermediate states, because of the hindered mobility of the components (due to viscosity or crosslinking).

\subsection{Phenomena in thin polymer films} Confining polymer blends in thin films preparation of the films and the presence of substrate/film and film/air interfaces. Some of the phenomena in polymer blends confined in thin films also occur in pores and therefore deserve description before focusing on confinement in AAO. Thin films of polymers are usually prepared by spin-coating or dipcoating. Each of these techniques possesses its specificity, as described below.

In spin-coating, one drop of a homogeneous polymer solution of polymer or polymer blend is deposited onto the substrate and spun at high speeds. The phenomena occurring during spin-coating have been studied by several authors. ${ }^{76,78-80}$ During coating, the phenomena taking place are solution spreading, wetting or dewetting, phase separation, solvent
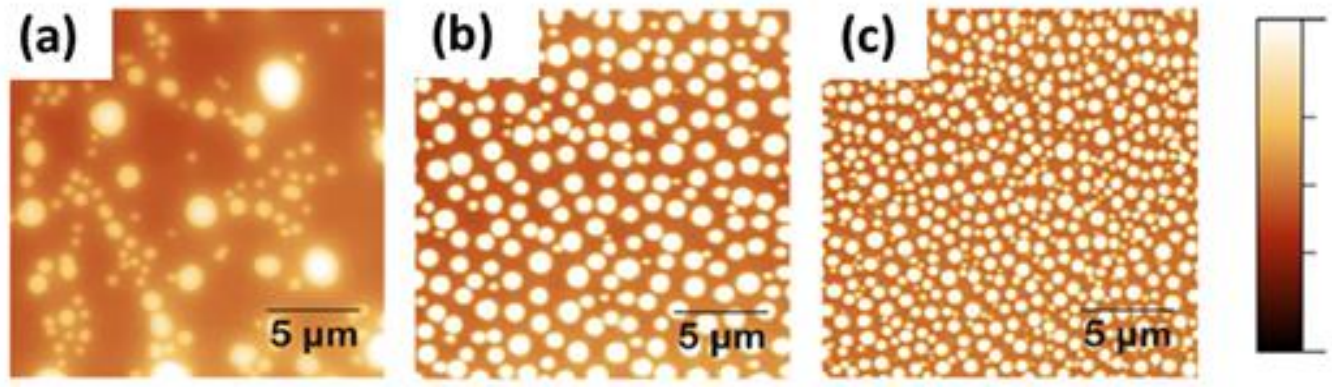

Fig. 3. Thin films of polymer blend obtained by dip-coating: AFM images of films of PS/poly(lactic acid) (PLA) blend obtained from a solution in DCE for three different conditions of dip coating ((a), (b), (c)). Z scale is -150 to $+150 \mathrm{~nm}$ for image (a) -50 to $+50 \mathrm{~nm}$ for images (b) and (c). Reproduced from Applied Surface Science 393, $127^{82}$, Copyright 2017, with permission from Elsevier.

introduces an additional complexity compared to the bulk due to the large number of superposed and interfering phenomena taking place during the evaporation, polymer deswelling, and vitrification. The substrate/solvent affinity is a determining factor in the spreading/wetting-dewetting of the polymer solution. 
For polymer blends, the component of lower energy tends to migrate to the film/air interface and the component of better affinity with the substrate tends to migrate to the film/substrate interface. ${ }^{81}$ During evaporation, the solubility of the polymers with the casting solvent is the determining factor for blends where the polymer with higher solubility enriches the film surface. The morphology can evolve from vertical stratification to a lateral domain structuring due to the interfacial instability originating from solvent concentration gradients (via a Marangoni-like instability). Solvent evaporation also leads to the loss of chain mobility down to the $T_{g}$ where the system is vitrified.

In dip-coating, the substrate is dipped into a polymer solution and removed at constant speed, leading to a thin film after solvent evaporation. In these procedures, a homogenous solution of polymer (or polymer blend) is deposited onto a substrate. During dip-coating, various phenomena are also superposed such as wetting/dewetting, phase separation, solvent evaporation, capillary flow, and viscous drag. As demonstrated by Vital et al. ${ }^{82}$ and illustrated in Fig. 3, the final morphology depends on many experimental parameters such as withdrawal speed, polymer blend composition, and solvent nature.

In both spin-coated and dip-coated thin films, the rapid evaporation of the solvent often traps the films in a non-thermodynamic state. A common method to reach a more stable state is to proceed to post-treatment, either solvent vapor annealing (SVA $)^{83}$ or thermal annealing. During these annealing procedures, the polymer is in a mobile state and reorganization can occur due to the same phenomena as those encountered during thin film preparation (i.e. via wetting/dewetting, phase separation, polymer migration, solvent evaporation). In the course of annealing (thermal or solvent vapor), the substrate often dominates the morphological evolution of the blend because the blend components are likely to have different wetting tendencies on the substrate and from each other. Binary polymer blend films can exhibit smooth (stratified) or rough surfaces, bicontinuous or discrete patterns, and stratified or perforated layers depending on the experimental conditions. ${ }^{84-85}$

To conclude, the final morphology of the films of polymer blends results from the interplay of numerous phenomena (wetting/dewetting, phase separation, swelling/deswelling, capillary flow, viscous flow, and vitrification) which relative importance are driven by parameters such as film thickness, FloryHuggins parameters between polymers and solvent, interfacial energies between polymers, substrate, air, and solvent, molar mass of polymers, conditions of film preparation and those of post-treatment if there is any. We will encounter these phenomena again as 
they are also prominent in establishing the morphology of polymer blends confined in AAO.

\subsection{Polymer blends in AAO}

When immiscible polymer blends are are formed. In the case of infiltration of a polymer solution, the situation is comparable to what we have previously described in the case of thin films, with a paramount influence of wetting/dewetting, capillarity,

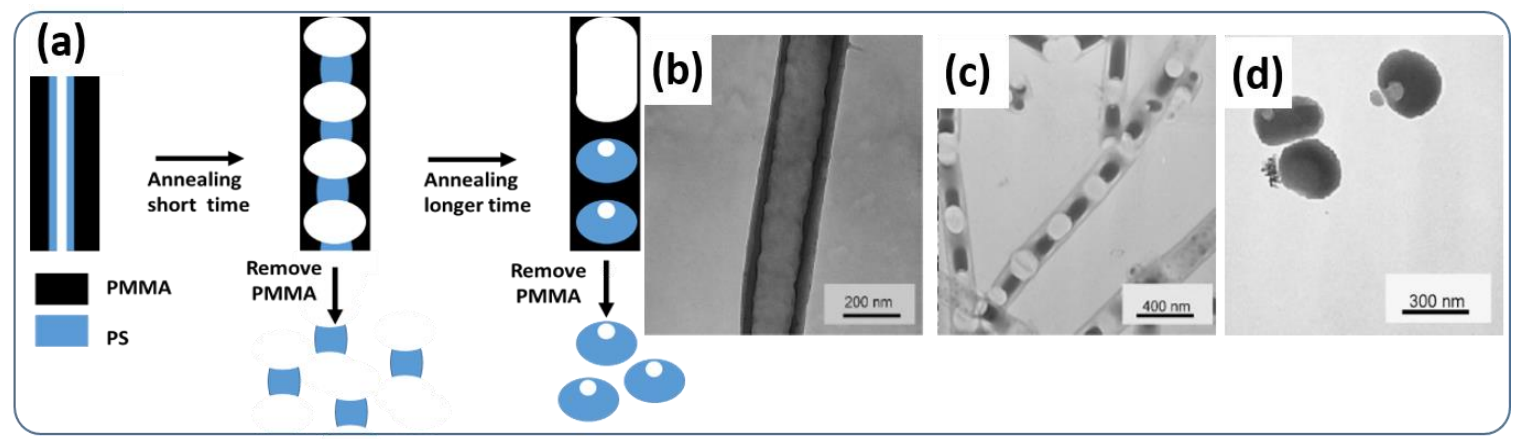

(e)

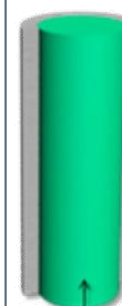

PS/PMMA in DMF

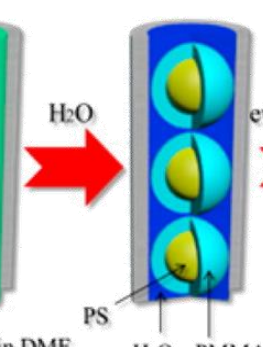

H2O PMMA

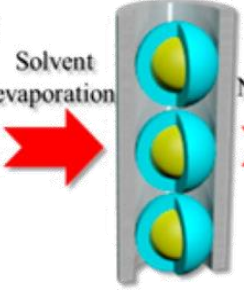

\section{(f) \\ (f)}

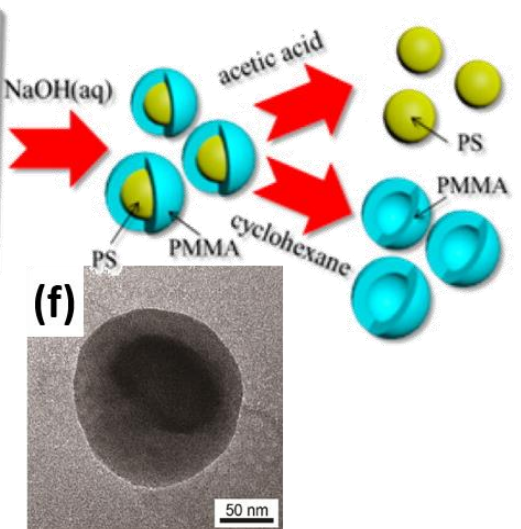

Fig. 4. Morphologies induced by surface affinity with the pore walls: Top: schematic of the evolution of a PS/PMMA blend confined in AAO as nanotubes by thermal annealing (a); Transmission Electron Microscopy (TEM) images of a nanotube (PMMA outer layer, PS inner layer) (b); (PMMA/PS rods with encapsulated air-pockets after thermal annealing at $190^{\circ} \mathrm{C}$ for $5 \mathrm{~min}(\mathrm{c})$; PS nanospheres after thermal annealing at $190^{\circ} \mathrm{C}$ for $8 \mathrm{~h}$ and removing PMMA for $8 \mathrm{~h}$ (d). (Note that for (b), (c), and (d) PS is stained). Reproduced from Macromol. Rapid Commun. 30, 37786, Copyright 2009, with permission from Wiley. Bottom: schematic of the evolution of a PS/PMMA blend confined in AAO by introduction of a non-solvent (water) in the confining porosity leading to PS core-PMMA shell nanospheres (e); TEM images of a nanosphere (f); a nanosphere after PMMA removal (g); Scanning Electron Microscopy (SEM) image of a nanosphere after PS removal (h). (Note that for (f) and (g) PS is stained selectively), Reproduced from ACS Macro Lett. 4, 71763, Copyright 2015, with permission from the American Chemical Society. 
importance and associated parameters are, however, not the same and will be detailed through selected examples in this section. In melt infiltration, the effect of the viscous flow also needs to be considered in addition to the previous parameters. Instead of a conventional presentation separating solution and melt infiltrations, we have organized this section into three different parts. Each one deals with the strategy followed to achieve the formation of heterogeneous morphologies in a controlled manner, namely: i) surface affinity with the pore walls, ii) viscous flow, and iii) sequential infiltration.

\subsubsection{Morphology induced by} surface affinity with the pore walls

Exploiting the differential affinity of the components of the blends (polymers and solvents) toward the walls of the AAO pores is a very common way to induce surface-induced phase separation in a homogenous solution of a polymer blend. It can result in a core-shell morphology, or more complex morphologies because of Rayleigh instabilities during infiltration or post-treatment. ${ }^{56,86}$ Rayleigh instability arises from the surface tension of a cylindrical system aiming to minimize the surface area by generating single droplets. ${ }^{87}$ The interested reader is referred to the work by Rayleigh ${ }^{88}$ and Lee et al. ${ }^{89}$ on both the history and application of the Rayleigh instabilities concept in polymer science. ${ }^{24}$
In the work of Chen et al. ${ }^{86}$, a good example of such behavior was shown (Fig. 4(a-d)). A solution of PMMA/PS in chloroform was infiltrated into AAO to form nanotubes (Fig. 4(b)). The preferential interaction between PMMA and AAO walls led to nanotubes with outer and inner layers of PMMA and PS. The phase separation between PS and PMMA takes place in the membranes and is governed by the AAO walls. This work also showed how more complex morphologies can be achieved by further posttreatments. For this purpose, the effect of thermal annealing above $\mathrm{T}_{\mathrm{g}}$ of these nanotubes was studied more specifically. Similar to the case of only PMMA homopolymer, the PS inner surface of the bilayer tube undulated and the instabilities grew in amplitude with a wavelength characteristic of Rayleigh instabilities. Through this treatment, a layer of PMMA remains at the wall surface and induces the creation of holecontaining PMMA/PS nanorods (Fig. 4(c)) with ultimately the formation of encapsulated PS nanospheres with small air pockets inside (Fig. 4(d)).

In a slightly different approach, Ko et al. ${ }^{63}$ exploited the selective affinity of the pore walls towards the solvent to form spherical core/shell nanoparticles (Fig. 4 (e-g)) using a double solution wetting method. The pores were filled with a PS/PMMA solution in DMF, the solvent of both PS and PMMA. The introduction of water, a non-solvent of the polymers, in a second step forms spherical PS core and 

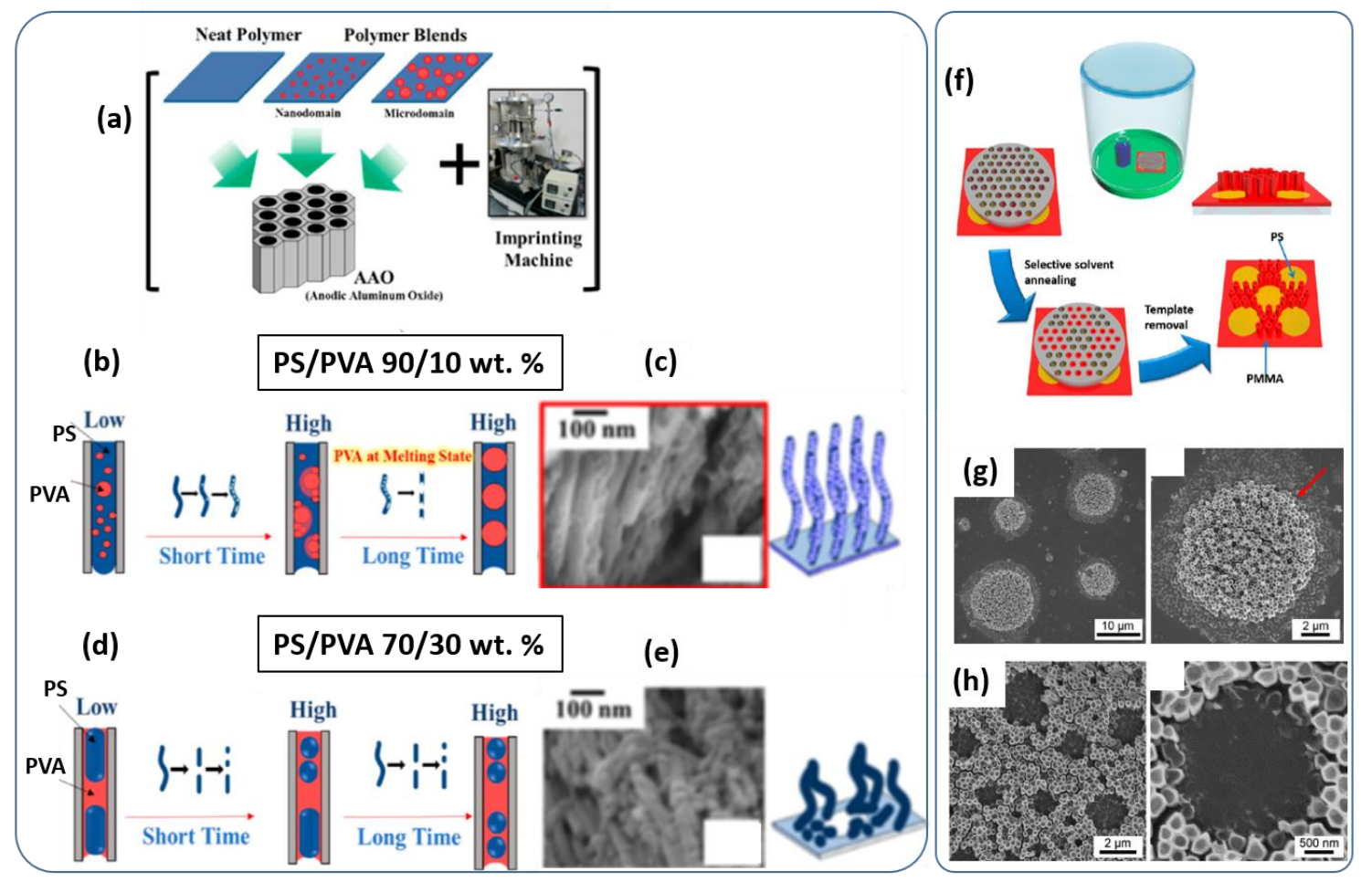

Fig. 5. Heterogeneous polymer blend infiltration leading to the fabrication of hierarchical nanostructures: Top: schematic of the process by thermal imprint of polymer blends (a); schematic of the evolution as a function of temperature and time (b), (d); SEM images (c), (e) of the resulting morphology for PS/PVA 90/10 wt. \% (b) (c) and PS/PVA 70/30 wt. \% (d) (e) Reproduced from Polymers 11, 1039-190, Copyright 2019, with permission from MDPI. Bottom: schematic of the process by the SAINT method for a PMMA/PS blend using a selective solvent of PMMA (f); SEM images for PS/PMMA 80/20 wt. \% (g); and 34/66 wt. \% (h) Reproduced from Langmuir 32, 2110¹, Copyright 2016, with permission from the American Chemical Society.

PMMA shell nanospheres (Fig. $4(f-g))$. This transformation driven by Rayleigh instability is due to the greater affinity of water to AAO than PMMA.

In melt infiltration, one generally deals with a heterogeneous viscous fluid impregnation (i.e. the polymers are already phase-separated when entering the pores). This differs from the solution wetting previously described, where the infiltrating medium is homogenous and phase separation occurs during infiltration. The final morphologies differ, and the core-shell morphology is not a common feature. A good illustration of this approach is the work by Muanchan et al. ${ }^{90}$ studying PS / poly(vinyl alcohol) (PVA) blended by the melt-mixing high-shear procedure (Fig. 5(a-e)). Innovatively, the nanostructures were prepared by the thermal imprint of the blends into AAO templates $(50,100 \mathrm{~nm}$ pore diameters). The PS/PVA 90/10 wt.\% blend exhibits a phase separation with a PS-rich matrix and PVA-rich nanodomains (10-30 nm). The affinity of PVA to AAO 
induces a surface-induced phase separation and the formation of PVA droplets at the surface of PS nanorods (Fig. 5(b-c)).

PS/PVA 70/30 wt.\% exhibits in thin film a phase separation with a PS-rich matrix and PVA-rich microdomains $(300 \mathrm{~nm}-30 \mu \mathrm{m})$, i.e a scale that is higher than the pore size of the AAO membrane. After infiltration and removal of PVA, PS nanorod arrays were observed in place of the PS phase (PS main component, PVA minor component). In place of the PVA phase (PVA major component, PS minor component), microholes were observed with nanorods and nanospheres of PS. In the latter case, the formation of PS nanostructures was attributed to Rayleigh instability inside the AAO pores (Fig. 5(d-e)). The differential affinity between the polymer and the solvent was also exploited to generate a selective SAINT infiltration in the case of a macroscopically phase-separated blend of PS and PMMA (Fig. 5(f-h)). ${ }^{91}$ An AAO membrane was placed onto a macro-phase separated film of a binary blend of PS and PMMA (Fig. 5(f)). Since the heterogeneity scale in the film is higher than the pore size of the AAO membrane, a selective infiltration of the PMMA into the membrane is obtained while PS remains outside the membrane using PMMA selective solvent vapors (acetic acid). After AAO dissolution, a hierarchical polymer film with PS domain and PMMA nanotubes was obtained (Fig. 5(g-h)).

\subsubsection{Morphology induced by}

\section{viscous flow}

Upon melt infiltration, a pronounced viscosity difference between the components of the blend can lead to morphological consequences. A good illustration of this behavior is the work by Tian et al. ${ }^{92}$ focusing on the infiltration of AAO with a heterogeneous blend melt composed of a hyperbranched polyester (HBP) and a linear PMMA polymer (L-PMMA) (Fig. 6(a-c)). As in the study by Ko et al. described above ${ }^{91}$, the heterogeneity scale in the film is higher than the pore size of the AAO membrane, preventing the two components (HBP and L-PMMA) from entering simultaneously into the same pore. Hence, distinct nanotubes of HBP on the one hand, and L-PMMA, on the other hand, are formed. The marked viscosity difference between the two components leads to differential kinetics of infiltration yielding long nanofibers for HBP and short nanorods for L-PMMA (Fig. 6(a-c)). After AAO etching, the polymer surface is composed of an array of HBP nanofibers and L-PMMA nanorods, and its characteristics such as wettability and droplet impact can be tuned by changing the number of terminal hydroxyl groups of HBP. 

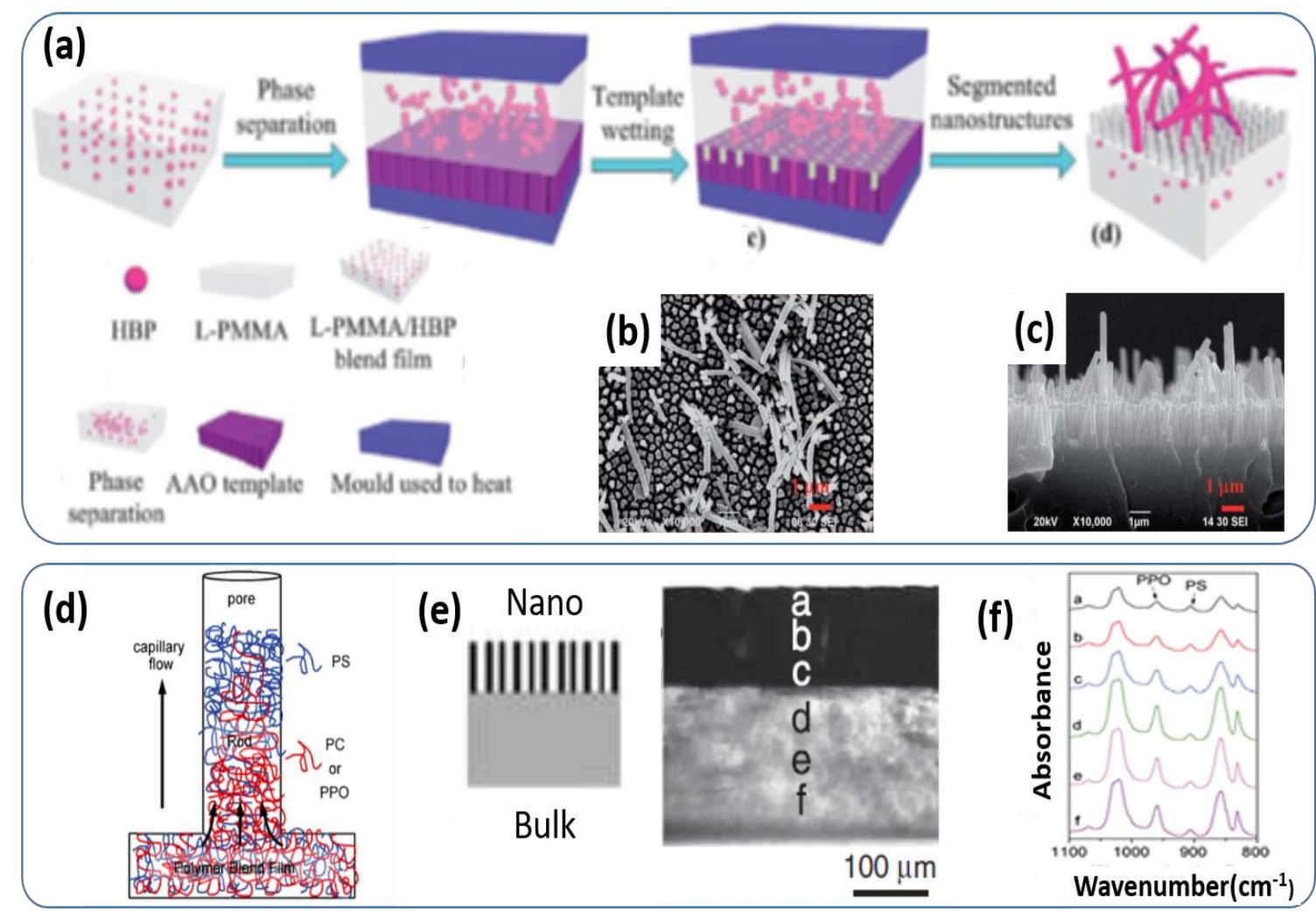

Fig. 6. Morphologies induced by viscous flow: Top: schematic of the fabrication process of nanostructures based on an L-PMMA/HBP polymer blend (a), SEM top-view (b), and cross-section images (c) of the nanostructures. Reproduced from RSC Adv. 4, 5302192, Copyright 2014, with permission from the Royal Society of Chemistry. Bottom: schematic illustration of gradient composition due to viscosity difference (d), optical micrograph and scheme of a nanorods/film blend (e), micro - Fourier Transform Infrared (FTIR) spectra for PPO/PS (f) infiltrated in $65 \mathrm{~nm}$ pores. Reproduced from Sci. Chi. Chem. 55, 726 $6^{94}$ Copyright 2012, with permission from Springer.

In homogeneous polymer blends, the differential viscous flow of the components can be exploited to generate heterogeneous structures inside the pores. In a series of papers, $\mathrm{Wu}$ and coworkers ${ }^{22}$, 93-95 examined the melt infiltration of AAO pores with miscible binary blend melts (50/50 wt.\%) of (poly(2,6dimethylphenylene oxide)/polystyrene (PPO/PS)) and (poly (2,6-dimethylphenylene oxide)/isotactic polystyrene (PPO/iPS)). The melt infiltration was performed by placing an AAO membrane on top of the polymer blend film, followed by thermal annealing above $250^{\circ} \mathrm{C}$. Nanorods were formed in all cases and FTIR analysis demonstrated that the PPO contents in the rods decreased from bottom to top (Fig. 6(d-f)). In the case of a pore diameter of $65 \mathrm{~nm}, \mathrm{PPO}$ content was $42 \%$ at the bottom of the rods, and only $28 \%$ at the top of the rods. PPO contents also decreased with the pore diameter. For the iPS/PPO blend, a gradient of 

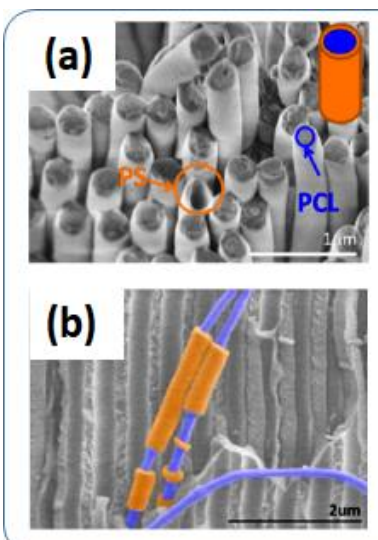

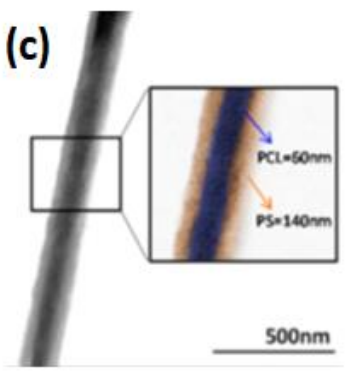

(d)

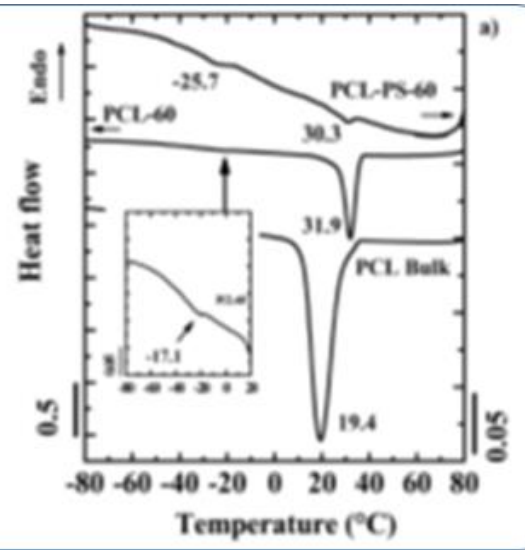

Fig. 7. Morphology induced by sequential infiltration: PCL-PS core-shell nanorods SEM top-view image (a) side-view image (b), TEM image(c), DSC cooling scan (d). Reproduced from Langmuir 32, 786099, Copyright 2016, with permission from the American Chemical Society.

crystallization was observed along the rods with a preferential orientation of the nanocrystallites with a c-axis perpendicular to the long axis of the nanopores. When the polymer blend enters the nanopores during capillary flow, its molecular composition distribution is governed by the viscosity gap between the polymers (PPO is much more viscous than PS) and the degree of confinement dictated by the pore diameter. The differential kinetics of penetration of the chains into the pores is thus governed by the molar mass of the polymer. This was demonstrated by Yao et al. ${ }^{96-97}$ studying the imbibition of melt blends of poly(ethylene oxide) PEO exhibiting long (500k) and short (50k) chains. Reflection microscopy measurements showed that short chains penetrate faster into the pores, inducing an enrichment of the short chains in the pores.

\subsubsection{Morphology induced by}

\section{sequential introduction}

In a completely different approach, the sequential introduction of two polymers into nanopores was shown to design heterogeneous nanostructures. ${ }^{56,} 86, \quad 98$ Hence, core-shell nanostructures can be formed. Dougherty in 2009 investigated the fabrication of core-shell (PMMAPLLA) nanotubes with first a solution infiltration of PMMA (to produce hollow tubes) followed by melt infiltration of PLLA. ${ }^{98}$ Variation of the thickness of PMMA shells was obtained by changing the concentration of the solution of PMMA. ${ }^{98}$ Similarly, Chen et al. ${ }^{86}$ also used a double infiltration method to prepare core-shell PS-PMMA nanorods, but with solution infiltrations for both PS and PMMA. Core-shell nanocylinders can also be obtained through a double infiltration in the melt. Poly $(\varepsilon-$ caprolactone)(PCL)(core)-PS(shell) nanocylinders were 


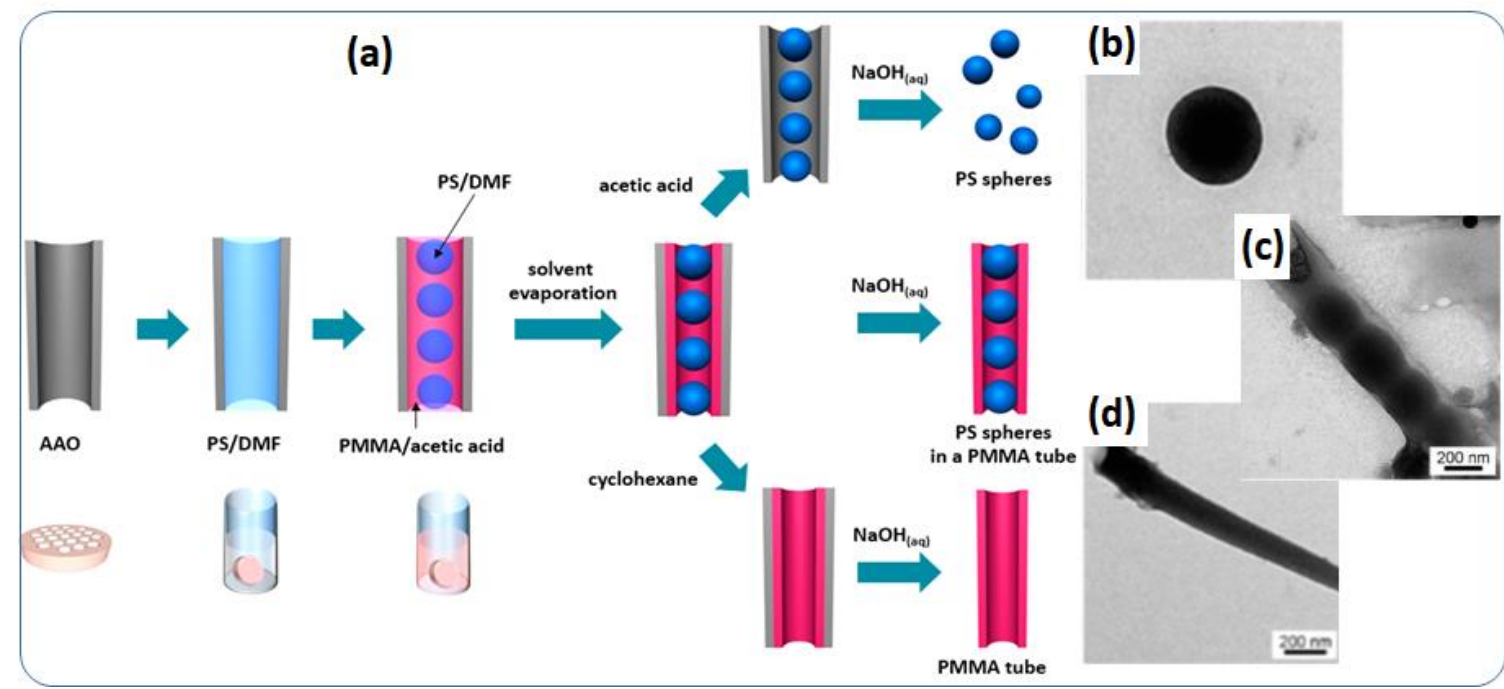

Fig. 8. Combination of sequential infiltration and differential affinity towards the pore walls: Schematic of the fabrication process of nanostructures based on PS/PMMA (a), TEM images of a PS nanosphere (b), PS nanospheres in PMMA tube (c) and PMMA tube (d). Reproduced from Macromolecules 47, 522756, Copyright 2014, with permission from the American Chemical Society.

obtained this way by Sanz et al. ${ }^{99}$ (Fig. 7). The first infiltration of PS (to produce hollow polymer nanotubes of PS) followed by a second infiltration of PCL forms the desired core-shell structure. Because the $T_{m}$ of PCL is lower than the $T_{g}$ of PS, PCL wets PS polymer nanotubes. As $\mathrm{PCL}$ is surrounded by the PS surface, this technique makes it possible to prepare samples to study the influence of the surface nature on the crystallization of the PCL fibers and to study the intrinsic properties of the materials confined in the core with different wall properties. When confined in the AAO template, PCL exhibited a fractionated crystallization at $31.9^{\circ} \mathrm{C}$ and $-17.1^{\circ} \mathrm{C}$. Confined in the PS nanotube, the temperature shifted to $31.2^{\circ} \mathrm{C}$ and $18.2^{\circ} \mathrm{C}$. The lowering of the lowest peak is attributed to defect-free crystallization due to the nature of the surface - PS versus AAO.

Moreover, the sequential introduction can be combined with the exploitation of the differential affinity of the components of the blends towards the pore walls of the AAO to generate even more complex nanostructures. Peapods were achieved by ${ }^{56}$ using a double solution wetting method (Fig. 8). A solution of PS in DMF is first introduced into the AAO nanopores and the second solution of PMMA in acetic acid is then introduced. Due to the preferential interaction between PMMA and AAO walls, PS is isolated in the center of the nanopores and after evaporation, nanospheres or nanorods of PS are formed depending on the PS concentration and $\mathrm{M}_{\mathrm{w}}$. A supplementary 
step consisting of water immersion and further evaporation leads to PS nanopods coated with PMMA as shown by the same group in 2015.100

\section{Block copolymers under confinement}

\subsection{Introduction}

BCPs are made up of two or more chemically different blocks linked via covalent bonds. ${ }^{80,}$ 101-104 These blocks are thermodynamically incompatible, due to a combination of an unfavorable enthalpy of mixing and negligible mixing entropy per unit volume. ${ }^{80}$ However, the covalent bond between the blocks prevents the macro-phase separation, and favors instead a phase separation in the nanometer scale (between 5 and $100 \mathrm{~nm}$ ), generating for example for linear diblock copolymers diverse ordered morphologies $77, \quad 80,102$ including lamellae (L), hexagonally packed cylinders (C), body-centered-cubic spheres (S), and a complex networked phase called gyroid (G). The periodic microstructure of BCPs is driven by the Flory-Huggins parameters $(\chi)$ as well as by the molecular architecture varying from linearity to miktoarm and cyclic structures. ${ }^{101}$ A disorder-order transition to any microphase occurs by quenching a system of the proper composition. ${ }^{77,103,105-106}$ For both fundamental science and technological applications, the assembly of BCPs presents a high-throughput technique for establishing ordered patterns by bottom-up nano-generation ${ }^{107-108}$ such as cylindrical, spherical, or lamellar micro-domains. ${ }^{104}$ As a result,
BCPs are envisioned in many technological applications such as lithographic masks, photonic materials, and nanoporous membranes, to name but a few. The ability of BCPs to form ordered nanostructures makes them ideal materials to investigate the formation of morphological features inside a limited space such as in thin films $s^{108-109}$ or AAO templates. ${ }^{20}$ When at least one dimension of the confining space is comparable to the natural period of the $B C P^{\prime} s$ self-assembly, the ordering and crystallization of BCPs at the nanoscale may exhibit drastic differences compared to bulk behavior. Depending on its dimension, geometry, and surface selectivity, the confining space introduces several constraints including entropy loss and surfacepolymer interactions that produce profound modifications in the BCP phase diagram. Crystallization behavior such as crystal form, crystallinity, chain orientation, and crystallization kinetics can also be deeply impacted. After a summary of the salient features of BCPs under confinement (corresponding to the work carried out during the $2000-2010$ period), this section will review the most relevant advances on the subject, both for morphology and crystallization, over the last decade. Although we chose to focus on experimental work, a summary of the major advances in the field of simulation is provided in the first section. 


\subsection{Salient features of BCP confinement in hard}

templates

Placing BCPs inside a confined environment can form novel morphologies differing from the ones obtained in the bulk and thin films. This has generated a considerable number of studies, both experimental and numerical.9, ${ }^{9}$ 15-16 Generally speaking, the formation of novel morphologies and the transformation from one morphology to another depend on the strength (degree) of the confinement (i.e. the ratio between the natural period of the polymer $\left(L_{0}\right)$ and the diameter of the pores (D)) and the pore wall compatibility for the blocks. In such a situation, the enthalpy and entropy of the polymers are modified, leading to a modification of the phase diagram of the self-assembly process. The entropy, which is related to the extent of stretching, is thus driven by the confinement strength. For the latter, when the commensurability of the natural period with the pore dimension is not satisfied, the exaggerated amount of stretching (or compression) of the blocks favors the formation of new phases. The enthalpy change is driven by interaction with the pore walls. Below is a summary of these combined effects (entropy and enthalpy) on the phase behavior of the most common BCP morphologies (we provide a list of relevant references at the end of each chapter).

In the case of the lamellar system, pore walls already decorated with $-\mathrm{OH}$ and without any further surface modification (thus non neutral) favor the development of parallel layers with a repeat period along the confining tube axis. Both blocks wet the pore walls (without enthalpy penalty) favoring the formation of patterns similar to the bulk since there are no imposed conditions on the period. If the walls enhance its preference for one block, a wetting layer of that block will establish, favoring the building of concentric structures. It can even provoke transition towards morphologies not seen in bulk for these compositions, like toroid. ${ }^{12-13,110-111}$ Submitting cylinder-forming BCP to confinement in AAO results in a myriad of morphologies, distinct from the bulk, driven both by the diameter of the pores and the pore walls' affinity towards the blocks. Concerning the latter point, the asymmetry of the BCP will produce different morphologies depending on whether the shortest or the longest block displays a selective affinity towards the walls. The variations in morphologies observed in this way are consequently larger compared to lamellae-forming BCPs, including helices (single and double), stacked toroids, multiple cylinders parallel or perpendicular to the pore axis, and even concentric perforated lamellae structures (for compositions near the cylinder-gyroid phase boundary). ${ }^{12-13,110}$ For gyroid-forming BCPs (the most complicated morphology in diblocks, containing two interpenetrating tri-functional networks in an array), 
the result is again dependent on the pore dimension and the selectivity of the pores towards the majority or the minority block: concentric perforated lamellae, helices, stacked toroids or even lamellae can be observed because the gyroid structure occupies a very narrow window between the lamellae and the cylindrical region. ${ }^{11}$

For sphere-forming diblock copolymers, the spherical structure is preserved and in the case of larger pores, forming spherical domains aligned along the pore axis. In contrast, it transitions towards new morphologies in pores with smaller diameters, such as core-shell cylindrical morphologies, single columns of spherical microdomains, and spirals of doubly and triply paired spherical micro-domains were observed. ${ }^{13}$

A large number of various polymer nanostructures observed experimentally in confined geometry motivated several researchers to conduct theoretical studies to model different morphologies of BCPs within a cylindrical pore. Simulation results might verify theoretical predictions. Simulative works could also give beneficial insights for potential experimental studies. ${ }^{112}$ Simulations on confined polymers could be applied to mimic a real-life problem as well. For example, Ha et al. ${ }^{113}$ he phase behavior of disk-coil $B C P s$ in confinement to gain insight into the selfassembled structure of chlorosomes that might manage its photosynthetic activities.
The main parameters that were varied in performing theoretical studies were the ratio of the periodic structure to the pore diameter and the nature of the pore wall surface energetics compared to those of the blocks of the BCP of interest. ${ }^{15}$ The contributions on BCPs confined to cylinders included Monte Carlo (MC) simulations ${ }^{112}$ and dissipative particle dynamics (DPD) studies. ${ }^{114}$ Simulations and theoretical studies predicted that depending on the neutrality or selectivity of the cylindrical pore walls, the confined polymers could show different morphologies: i) perpendicular lamellae or stacked disks in neutral walls, and ii) concentric lamellae for symmetric diblock copolymers inside strongly selective surfaces. ${ }^{16}$

Among the theoretical approaches, dynamic density functional theory (DDFT) simulations found kinetic pathways that confined lamellar BCPs take before reaching the equilibrium structures ${ }^{115}$, while DPD simulations can mimic laboratory conditions such as the flow of polymers into the nanopores in the course of the wetting period. ${ }^{116}$ by Li et al. ${ }^{117}$ suggested that self-consistent field (SCF) simulations could provide thermodynamic insights into the borders of stability regions of the polymeric nanostructures generated. According to a study by Zhu and Jiang ${ }^{118}$, MC simulations could be complementary in designing novel polymeric nanomaterials in the confined space. 


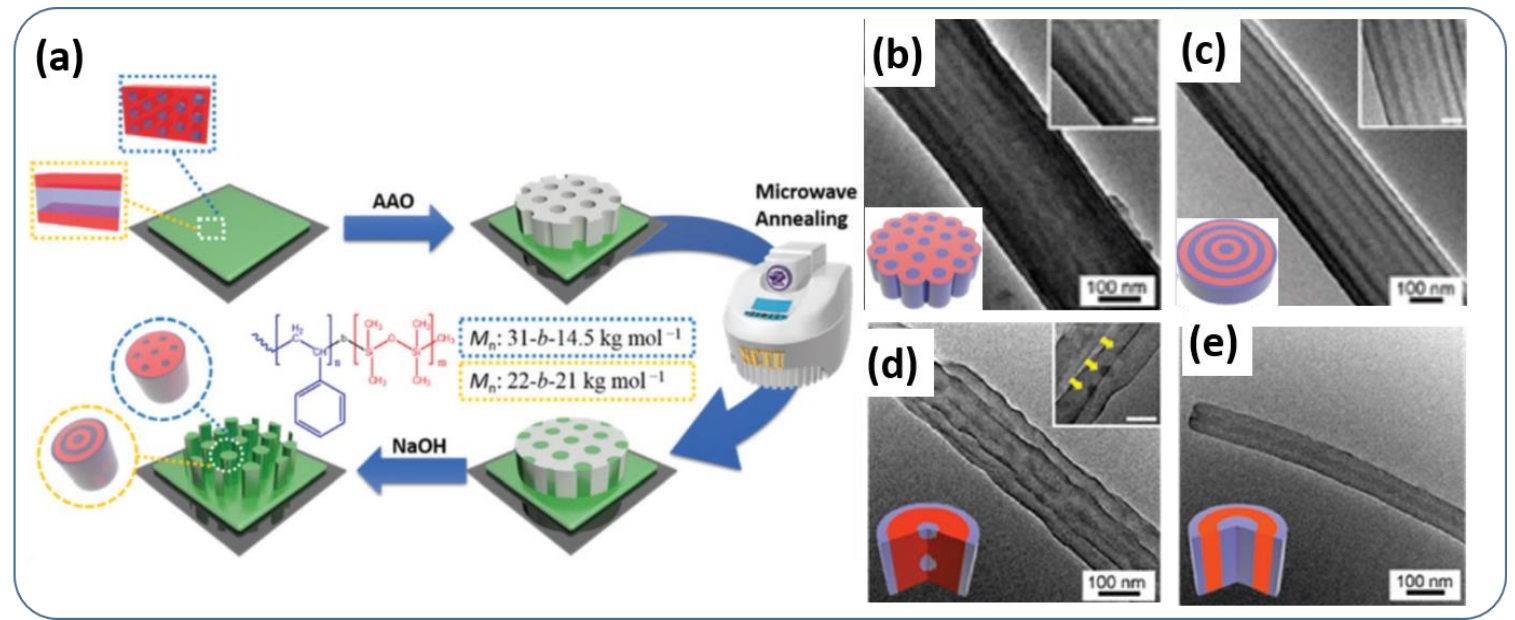

Fig. 9. Microwave-annealing infiltration: Schematic of the fabrication process of nanostructures based on PS- $b$-PDMS (a); TEM images and graphical illustrations in inserts (PS in red, PDMS in blue) of PDMS cylinder-forming (b); lamellaforming block polymer nanorods (c) confined in $240 \mathrm{~nm}$ pores; PDMS cylinder-forming (d) lamella-forming (e) block polymer nanorods confined in $60 \mathrm{~nm}$ pores. Reproduced from Soft Matter, 14, 3567, Copyright 2018, with permission from the Royal Society of Chemistry.

\subsection{Recent advances in confined BCP}

The behavior of simple BCPs in a confined environment was already mostly deciphered thanks to the great deal of work produced in the $2000-2010$ period, as described above. In the last decade between 2010-2020, innovations mostly concerned the use of a greater variety of infiltration processes of the BCPs, benefiting from the progress made in the case of infiltration of homopolymer (microwave, SAINT, double infiltration method). Blends of BCPs and homopolymers were also used to explore new behaviors. In comparison, the case of the triblock emerged in the last decade but remained rather confidential.

\subsubsection{New infiltration processes}

\section{and post-treatments}

Microwave annealing initially developed for homopolymers was successfully used to infiltrate BCP melt into the nanoporous AAO templates and provoke microphase separation. The phase behavior is dominated by the interaction with the pore walls and dimensions of the pores, similarly to more conventional procedures of solution or melt infiltration. For example, PDMS cylinder-forming and lamella-forming poly(styrene-block-dimethylsiloxane) (PS-b-PDMS), respectively, with a period of 34.9 and $41.7 \mathrm{~nm}$ were infiltrated into nanoporous $A A O$ templates by microwave annealing (Fig. 9(a)). ${ }^{67}$ Since PDMS interacts more strongly than PS with the pore walls, PDMS always preferentially covers the surface of 
the nanostructures. Typically, confined morphologies composed of PDMS cylinders parallel to the pore axis and concentric lamellar morphology were observed in larger pores $(240 \mathrm{~nm})$ for cylinder-forming and lamella-forming PS-b-PDMS (Fig. 9(b-c)). For smaller pores $(60 \mathrm{~nm})$, a cylindrical morphology based on a core (PS with spherical domains of PDMS embedded on the central axis) and a shell (PDMS) was seen for cylinder-forming PS-b-PDMS (Fig. 9(d)). For lamellaforming PS- $b$-PDMS, the confinement in the smaller pores leads to a double core-shell morphology with the sequence of PDMS, PS, and PDMS (Fig. 9(e)). ${ }^{67}$ After its initial development for the infiltration of homopolymer, the SAINT method was successfully applied to infiltrate the BCP samples in AAO templates. In the SAINT approach, the solvent vapors enable the swelled polymer chains to wet the nanopores of the porous templates and mediate the interaction between the polymer segments and the pore walls, facilitating the control of the morphologies. Using different solvent vapors that can change the effective volume fractions of both blocks and the interaction with the pore walls enables the morphologies of the BCP nanostructures to be tuned. Very interestingly, this process combines infiltration and phase behavior control (as in SVA post-treatment), due to the presence of solvent vapor throughout the process. Compared to more classical infiltration methods, the SAINT approach consequently provides the potential to end up with a greater variety of morphologies with a given BCP composition. The potential of this approach was shown by Cheng and coworkers ${ }^{119}$ for a lamella-forming PS- $b$-PDMS with a period of $36 \mathrm{~nm}$ and AAO with a pore size of $150-400 \mathrm{~nm}$ using a solvent mixture of toluene and cyclohexane in different proportions, toluene being a better solvent for PS, while hexane is better for PDMS (Fig. 10(a)). Changing the composition of the solvent mixture, while changing the volume fraction of the two blocks PS and PDMS that increase the fraction of swelled PDMS, varies the morphologies to give a concentric lamellar morphology (Fig. 10(b)), the winding cylinder morphology (Fig. 10(c)), and the irregular hybrid morphology (Fig. 10(d)).

Similarly to the post-treatments traditionally used for controlling polymer thin film self-assembly by giving mobility to the polymer chains trapped in a kinetic metastable and ill-defined state in the formed thin film ${ }^{83}$, SVA can also be employed to drive (or modify) the morphology of BCPs confined in AAO templates. Despite the interesting perspective offered by such an approach, the number of studies in this area is scarce. A good example is provided by 


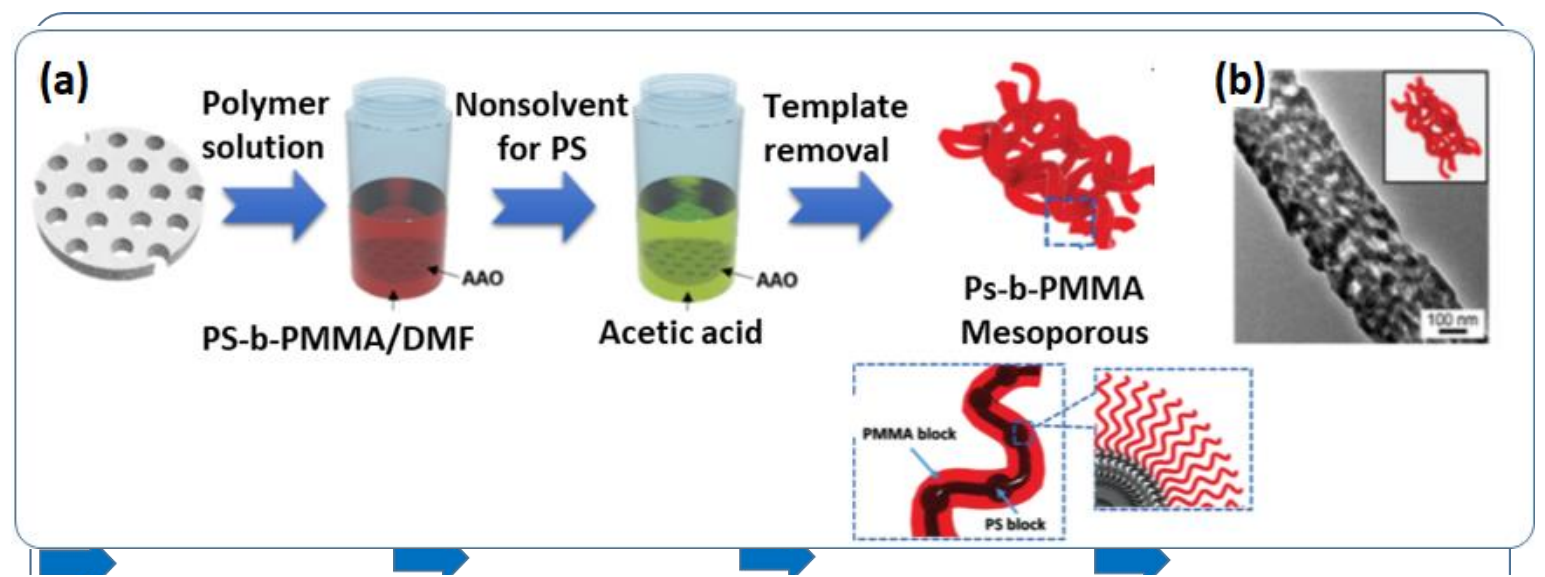

Fig. 11. Double solution wetting method for PMMA cylinder-forming PS- $b$-PMMA: Schematic of the method used (a), TEM micrographs and graphical illustration (PMMA in red and PS in dark blue) of the structures obtained (b). Reproduced from Polym. Chem., 8, 3399121, Copyright 2017, with permission from the Royal Society of Chemistry.
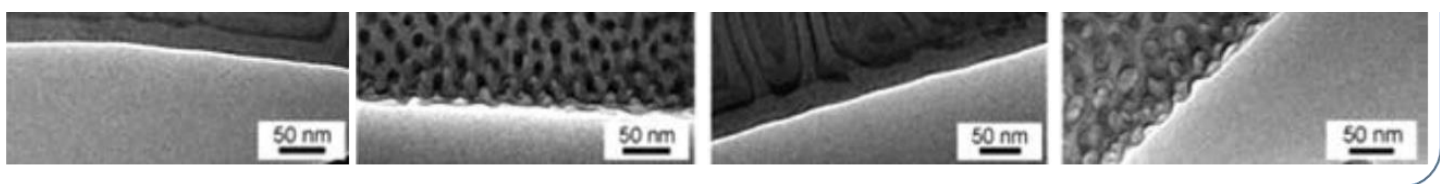

Fig. 10. SAINT and SVA methods applied to PS- $b$-PDMS block copolymer. Top: schematic of the SAINT method (a); TEM images and graphical illustrations in inserts (PDMS in purple and PS in yellow) of the nanorods obtained for lamellaforming PS- $b$-PDMS after annealing in a mixture of toluene/hexane vapor and HF solution treatment to remove PDMS for a toluene volume concentration $\left(\square_{\text {Tol }}\right)$ of 1 (b) and 0.16 (c) Reproduced from Soft Matter, 12, 8087 ${ }^{119}$, Copyright 2016, with permission from the Royal Society of Chemistry.

Bottom: Reversibility of the morphological transformation by SVA for a PDMS cylinder-forming PS- $b$-PDMS block copolymer: TEM images and graphical illustrations in inserts (PDMS in blue and PS in green) after annealing in a mixture of toluene/hexane vapor with $\square \square$ Tol $=0.14(\mathrm{~d}), \square_{\mathrm{Tol}}=1(\mathrm{e}), \square_{\mathrm{Tol}}=0.14(\mathrm{f}) \square_{\mathrm{Tol}}=1$ (g). Reproduced from Int. J. Polym. Mater. Polym. Biomater., 65, 695 120 , Copyright 2016, with permission from Taylor \& Francis.

Chu et al. ${ }^{120}$, demonstrating the reversibility of this morphological evolution in the case of cylinderforming PS-b-PDMS (Fig. 10(e-h)). Pure toluene as the annealing solvent results in the alignment of the cylindrical PDMS domains perpendicular to the AAO walls (Fig. 10(f, h)). When the solvent ratio of toluene decreases to 0.14 , the cylindrical PDMS domains are aligned parallel to the tube axis (Fig. 10(e, g)).
Repeated solvent vapor annealing processes can switch the morphologies. A difference in surface interaction between the swollen blocks and the pore walls is proposed to explain this behavior.

An evolution of the solution wetting method, the so-called "double solution wetting method" in which templates are dipped in two different solutions in sequential order, was used to 
manipulate morphologies and nanostructures of homopolymers and homopolymer blends. ${ }^{63,71-72}$ Using this approach, mesoporous nanotubes were prepared by a selective solvent-induced reconstruction in nanopores. $^{121}$ For that purpose, a sequence of experiments combining the infiltration of the $A A O$ template by a solution of the PMMA cylinder-forming PS- $b$-PMMA in DMF (a good solvent for both blocks), addition of acetic acid (a non-solvent for the PS, but miscible with DMF), subsequent evaporation of the solvents and dissolution of the template was used (Fig. 11(a)). When acetic acid is introduced into the nanopores containing the solution of the BCP in DMF, acetic acid can mix with the solution, causing a selective swelling effect on the PMMA blocks (and probably the precipitation of the PS). This effect causes the morphology reconstruction, forming worm-like mesoporous nanotubes after drying and elimination of the AAO template (Fig. 11(b)). The same group pushed forward this concept of non solvent-driven morphology to produce a greater diversity of nanostructures such as nanospheres (Fig. 12(a-e)). ${ }^{122}$

For that purpose, a PBD cylinder-forming PS- $b-\mathrm{PBD}$ solution in N-Methyl-2-pyrrolidone (NMP) was used to wet the nanopores. Nanotubes with PBD cylinders perpendicular to the pore walls were formed in the absence of non-solvent (Fig. 12(a,c)). When acetic acid (acting as the non-solvent) was added to the system, PS- $b$-PBD nanospheres were formed through a
Rayleigh-instability-type transformation mechanism

(Fig. 12(b,d,e)). PS- $b$-PBD/PMMA core/shell nanospheres were also fabricated using this strategy. 

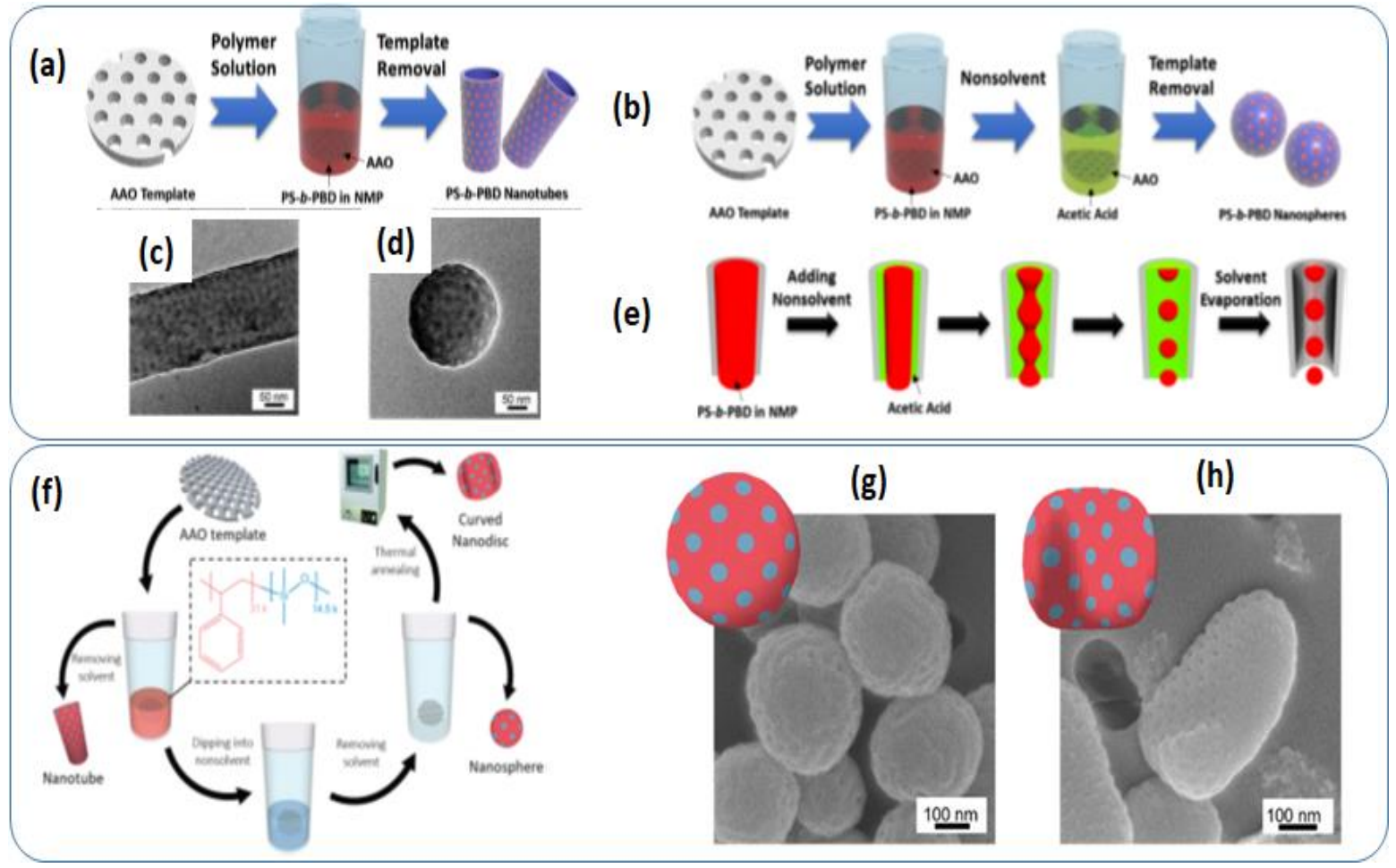

Fig. 12. Fabrication of nanoobjects using a double solution wetting method. Top: schematic of the fabrication process of PS- $b$-PBD nanorods using a single solution wetting method leading to nanorods (a), using a double solution wetting method leading to nanospheres (b) (PS in blue and PBD in red). TEM images of the PS- $b$-PBD nanorods (c) and nanospheres (d). Graphical illustration of the formation of nanospheres by Rayleigh-instability-type transformation (e). Reproduced from Langmuir 34, $14388^{122}$, Copyright 2018, with permission from the American Chemical Society. Bottom: schematic of the fabrication process of PS- $b$-PDMS nanostructures using a single solution wetting method (f). SEM images of porous nanospheres after removal of PDMS (g) and curved nanochips after thermal annealing (h). Reproduced from Soft Matter, $82,8201^{123}$, Copyright 2019 , with permission from the Royal Society of Chemistry.

Using the same double solution wetting approach, Lin and coworkers ${ }^{123}$ prepared PS-b-PDMS nanospheres by infiltrating first a PS- $b$-PDMS solution in toluene (a good solvent for both PS and PDMS) and subsequent dipping in a non-solvent mixture of acetic acid and ethanol (Fig. 12(f-g)). In comparison, PS- $b$ PDMS nanotubes were obtained via a conventional solution wetting with a PS- $b$-PDMS/toluene solution.
Thermal annealing of the PS- $b$-PDMS nanospheres above the $T_{g}$ of polymers in the AAO templates induces the formation of curved nanodiscs (Fig. 12(h)). Adjusting the annealing duration and temperature modified the characteristics of these curved nanodiscs. The transformation of PS- $b$-P4VP nanorods or nanotubes into nanospheres driven by Rayleigh instability can be achieved by a post-treatment 


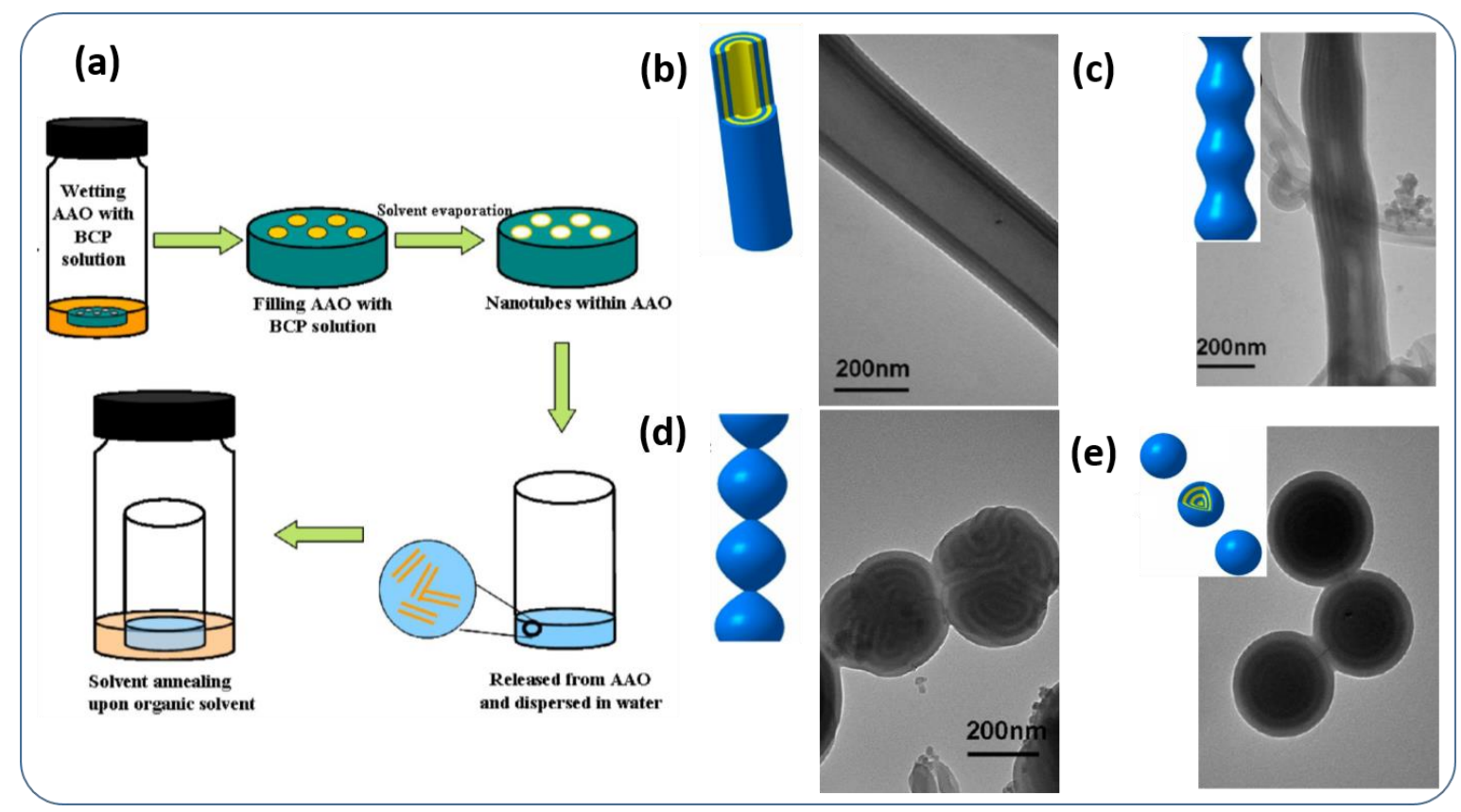

Fig. 13. Schematic of the fabrication process of PS- $b$-P4VP nanostructures with infiltration, release and post-annealing of the nanostructures (a). TEM images and graphical illustrations in inserts (P4VP in blue and PS in yellow) of a nanotube (b) and its transformation to nanospheres by solvent annealing for $10 \mathrm{~min}$. (c), $20 \mathrm{~min}$. (d), $180 \mathrm{~min}$. (e) (P4VP domains appear darker after staining). Reproduced from Langmuir 31, 1660124, Copyright 2015, with permission from the American Chemical Society.

consisting of vapor annealing of an aqueous

suspension of nanorods or nanotubes as demonstrated by Yan (Fig. 13). ${ }^{124}$ Apart from shape evolution, this "solvent absorption swelling" can also lead to morphology evolution depending on the volume fraction of blocks, annealing solvent nature, and time. Starting from a concentric lamellar cylindrical morphology and ending up with a concentric lamellar spherical morphology is the simplest example of this transformation (Fig. 13).

Tri-blocks can also be used in such a double solution wetting approach. Zhao et al. ${ }^{125}$ demonstrated that polymer nanotubes of PI- $b$-PS- $b$ -
P2VP inside AAO pores can evolve to cylindrical micelles, micelle bundles, open hollow tubes, closed hollow tubes, and finally to vesicles with a diameter equivalent to that of the pore diameter, by heating the infiltrated tri-block polymer/toluene solution in a selective solvent of P2VP, namely ethylene glycol (Fig. 14). A schematic of the vesicles is proposed with P2VP on the outer and inner wall of the vesicles and PS in between and PI in the core of the PS regions. 


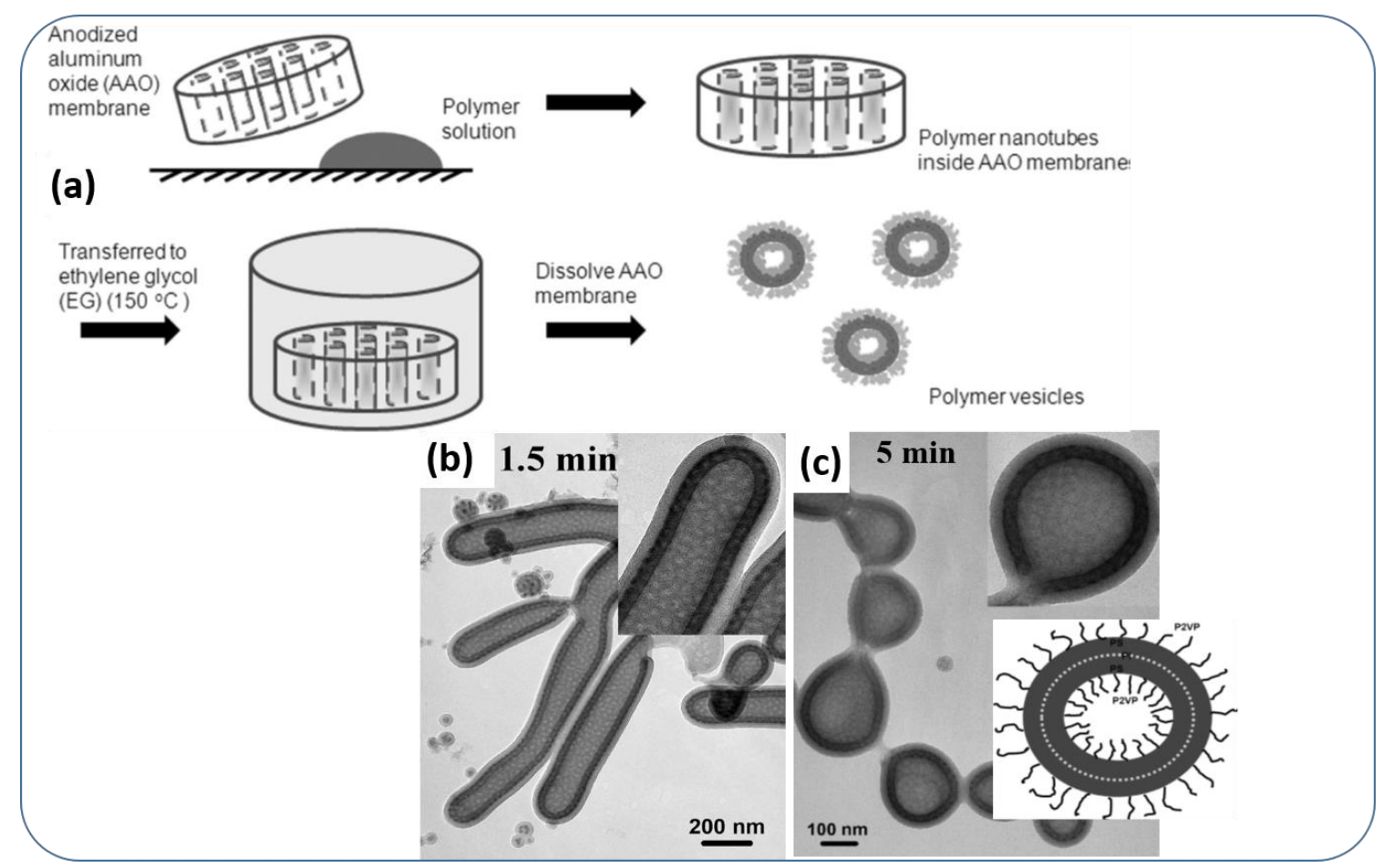

Fig. 14. Vesicles obtained using a double solution wetting approach for PI-b-PS- $b$-P2VP triblock copolymer: Schematic of the fabrication process (a), TEM images of vesicles obtained after triblock infiltration and after subsequent heating in a selective solvent of P2VP (PI is selectively stained) for $1.5 \mathrm{~min}$ (b), $5 \mathrm{~min}$ (c) (In insert a graphical illustration of the structure of the vesicle). Reproduced from ACS Nano 5, 486125, Copyright 2011, with permission from the American Chemical Society.

\subsubsection{Homopolymer-copolymer}

\section{blends}

As exemplified in the previous section in the SAINT method, one way to control the confined morphology is to change the volume ratio of the blocks by swelling specifically one block compared to the other. Another method to change the volume fraction of the blocks is to add a homopolymer and to confine a $\mathrm{BCP} /$ homopolymer blend in the $\mathrm{AAO}$ templates. Several authors used this strategy.

Cheng et al. in 2017 established a phase diagram (Fig. 15(a)) using lamella-forming PS-b-PDMS (43k) BCP blended with different amounts of homopolystyrene (hPS $(4.7,24$ or $820 k)$ ), infiltrated into the AAO templates (pore diameter of $150-400 \mathrm{~nm}$ with the SAINT method). ${ }^{126}$ Depending on the blending ratio (weight ratio between the $\mathrm{BCP}$ and the homopolymer) and the molecular weight ratio $(\alpha)$ between the homopolymer and the corresponding block in the copolymer, specific morphologies were obtained such as a multi-helical morphology (Fig. 15(b)) not observed in bulk or thin film. The molecular weight ratio $(\alpha)$ defines two regimes: a dry brush regime $\left(\alpha>>1, M_{\mathrm{hPS}}=820 \mathrm{k}\right)$ with the homopolymer 

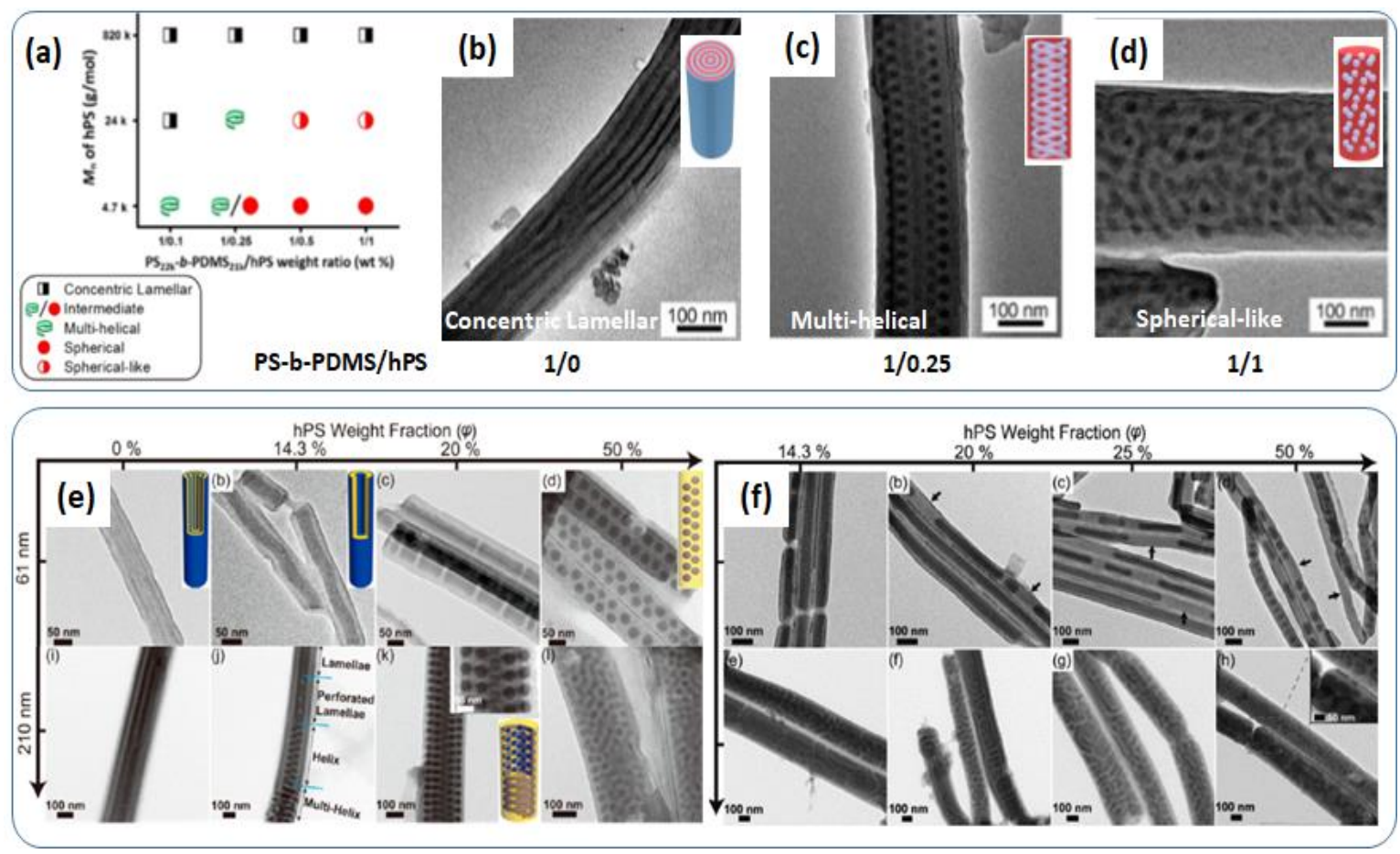

Fig. 15. Confinement of homopolymer-copolymer blends. Top: Case of lamella-forming PS- $b$-PDMS block copolymer blended with polystyrene (hPS). Morphology diagram for different weight ratios and hPS molecular weights (a), TEM images and graphical illustrations in inserts (PDMS in blue and PS in red) of the observed nanorods for PS22k- $b$ PDMS21k/hPS24k weight ratios of 1/0 (b), 1/0.25(c), 1/1(d). Reproduced ACS Appl. Mater. Interfaces 9, 21010 126 , Copyright 2017, with permission from the American Chemical Society. Bottom: Case of lamella-forming PS- $b$-P4VP block copolymer blended with polystyrene PS (hPS). TEM images and graphical illustrations in inserts (P4VP in blue and PS in yellow) of the observed nanorods as a function of channel diameter (61 and $210 \mathrm{~nm}$ ) and hPS weight fraction between 0 and $50 \%$ for an hPS molecular weight of $2.8 \mathrm{k}$ (e) and 21k (f). Reproduced from Langmuir, 31, 12354 ${ }^{128}$, Copyright 2015, with permission from the American Chemical Society.

tending to phase separate from the corresponding block, and a wet brush regime $\left(\alpha<<1, M_{h P S}=4.7 k\right)$ where the homopolymer is solubilized in the corresponding block. A molecular weight ratio of $1\left(\alpha=1, M_{h P s}=24 k\right)$ is an intermediate case, and increasing the blending ratio, in this case, leads to successively concentric lamellar (Fig. 15(b)) multi- helical (Fig. 15(c)), and spherical-like (Fig. 15(d)) morphologies.

The modification in the phase behavior compared to bulk and thin film by the confinement and the impact of the molecular weight of hPS was also demonstrated by Kim et al. ${ }^{127}$ on blends of lamellaforming poly(styrene-block-butadiene) (PS-b- 
PBD) (89k, a period of $42 \mathrm{~nm}$ ) (SBD) and polystyrene homopolymer (hPS (4.8 or $45 \mathrm{k})$ ) introduced and reorganized into AAO pores of $170 \mathrm{~nm}$ by wetting from a solution and thermal annealing. For low molecular weight hPS, hPS induced the structural transformation in such way as to yield more curved interfaces going from lamellar to helical and spherical morphologies, while the addition of higher molecular weight hPS $(\alpha=1)$ expanded the microdomains of PS up to the formation of a vesicular morphology on the center axis of the pores. The comparison of SBD/hPS blends in both bulk and confined states revealed that the constraints induced by the hard confinement significantly enhance the effect of hPS presence on the
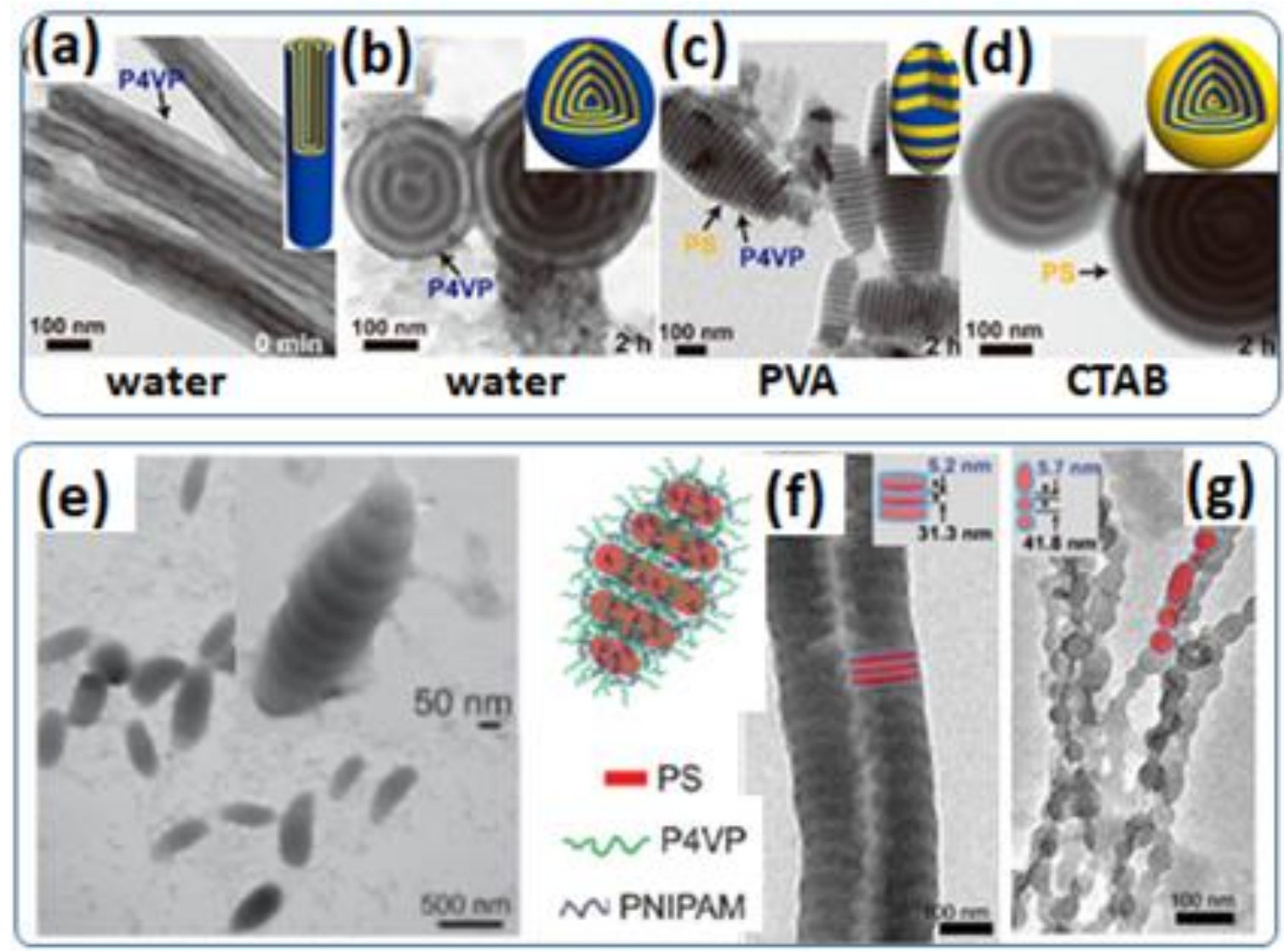

Fig. 16. Switch from 2D confinement to 3D confinement and vice-versa. Top: Morphological evolution of PS- $b$-P4VP nanorods in the solution annealed in solvent vapors: TEM images and graphical illustrations in inserts (P4VP in blue and PS in yellow) in water (a, b), PVA aqueous solution (c), and CTAB aqueous solution (d) annealed in chloroform vapors for 0 min (a), 2 h (b,c,d). Reproduced from Langmuir, 31, 12354128, Copyright 2015, with permission from the American Chemical Society. Bottom: Evolution of multicompartment micelles obtained via the self-assembly of PS- $b$-P4VP/PS- $b$ PNIPAM mixture in THF/water solution: TEM images and graphical illustration of the micelles before (e) and after confinement in AAO nanopores with a diameter of $254 \mathrm{~nm}$ (f) and $94 \mathrm{~nm}(\mathrm{~g})$. Reproduced from Phys. Chem. Chem. Phys., 22, $1194^{129}$, Copyright 2020, with permission from the Royal Society of Chemistry. 
phase transition of BCPs in the homopolymer/BCP blend. The addition of homopolymer led to the morphological transformation to compensate for the loss of conformational entropy of the polymer chains under 2D confinement.

The impact of the degree of confinement on the morphology was specifically addressed by $\mathrm{Xu}$ et al. ${ }^{128}$ in addition to the influence of the molecular weight $\left(M_{w}\right)$ of the homopolymer. Blends of poly(styrene)-block-poly(4-vinylpyridine) (PS-b-P4VP) ( 22 or $44 \mathrm{k})$ and homopolymer hPS $(2.8,21$ or $876 \mathrm{k}$ ) were introduced in nanoporous AAO templates (pore diameters of $61,96,210 \mathrm{~nm}$ ) by wetting from a solution and reorganized by SVA (Fig. 15(e-f)). In the wet-brush regime for low $M_{w}$ homopolymer $(\alpha=0.13)$ (Fig. 15(e)), a strong degree of confinement ( $D=1.7 \mathrm{~L})$ leads the system to evolve by increasing the homopolymer fraction from a concentric lamellar to a concentric cylindrical morphology, then the fragmentation of the cylinder results in spherical P4VP domains. With a weaker degree of confinement $(D=$ $5.7 \mathrm{~L}$ ) the evolution of the system goes through more original morphologies such as perforated lamellae, single helices, helices with chains of spheres, and finally spheres. A dry brush regime $(\alpha=40)$ (Fig. 15(f)) leads to macrophase separation and for the intermediate regime a competition exists between macro- and micro-phase separations, with the transformation of lamellar to spherical morphology for a strong degree of confinement and the coexistence of lamellar, cylindrical and spherical morphology for a weaker degree of confinement. The authors also used the morphologies developed with a PS- $b-\mathrm{P} 4 \mathrm{VP} / \mathrm{hPS}$ blend in AAO pores, especially the spherical ones to generate mesoporous materials. After removing the AAO membranes, the nanorods obtained were dipped into a selective solvent of P4VP. The swelling of P4VP in a selective solvent (ethanol) and collapsing the swelled P4VP generated mesoporous cylindrical materials with different pore sizes, form, and packing type. Solvent-absorption annealing (SVA of aqueous suspension) of the nanorods (Fig. 16(a)) with chloroform, a good solvent of both blocks, transforms them into nanospheres. This switch from a $2 D$ hard confinement into a 3D soft confinement is driven by Rayleigh instability, and the nanostructure and inner resulting morphology are controlled by the affinity of the water or water surfactant to the blocks (Fig. 16/a-

d)). Water preferentially wets P4VP, CTAB, PS, and PVA is almost neutral for PS and P4VP. Starting from concentric lamellar cylindrical nanorods, these differential affinities lead to onion-like spheres with a shell of P4VP for water (Fig. 16(b)), pupa-like nanostructures for PVA (Fig. 16(c)) and onion-like spheres with a shell of PS (Fig. 16(d)).

The switch from 3D soft confinement to 2D hard confinement was studied for a more complex 


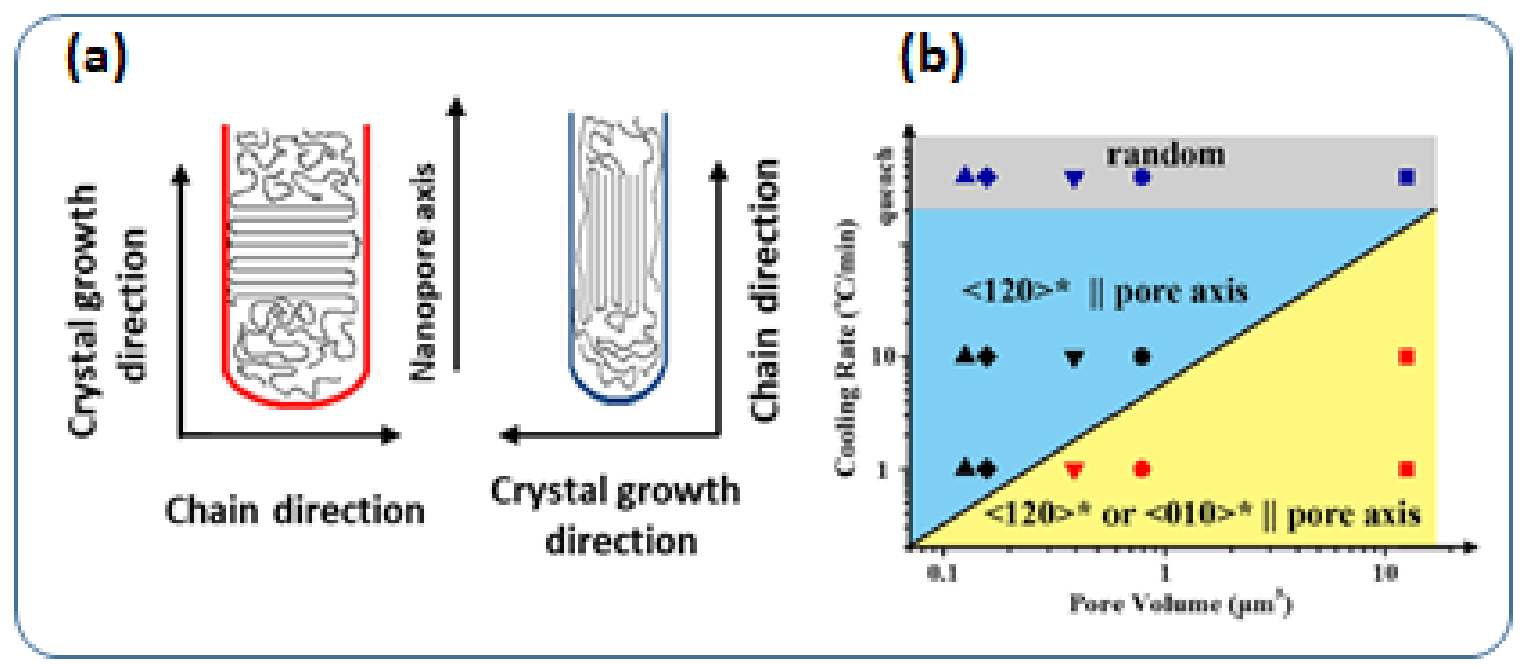

Fig. 17. Crystallite orientation with AAO nanopores: Schematic representations of the possible lamellar orientations within AAO nanopores (a). Reproduced from J. Polym. Sci. Part B: Polym. Phys., 52, 117917, Copyright 2014, with permission from Wiley. Diagram of the orientation mode of PEO infiltrated in AAO templates as a function of pore volume (400 nm-100 $\mu \mathrm{m}$ (squares), $100 \mathrm{~nm}-100 \mu \mathrm{m}$ (circles), $100 \mathrm{~nm}-50 \mu \mathrm{m}$ (down triangles), $100 \mathrm{~nm}-20 \mu \mathrm{m}$ (diamonds), and $40 \mathrm{~nm}-100 \mu \mathrm{m}$ (up triangles)) and cooling rate (b). Reproduced from Macromolecules 54, 9484 ${ }^{130}$, Copyright 2018, with permission from the American Chemical Society.

system, with a blend of two BCPs. Yue et al. ${ }^{129}$ examined the self-assembly of a blend of two BCPs: PS$b$-P4VP and PS- $b$-PNIMa, and the influence of the confinement of this blend in AAO nanopores (Fig. 16(e-

g)). In THF/water mixture, depending on the THF/water ratio, these two BCPs exhibit cooperative self-assembly leading to either small spherical assembly (SMA) or "pupa-like" multicompartment micelles (MCM) with an ellipsoidal shape (Fig. 16(e)). Upon 2D confinement, the copolymer blend exhibits a long defined MCM with a uniform diameter that the authors named long multicompartment micelles (LMCM). As the confinement degree increases with the decrease in the pore diameter, the LMCM is transformed into a polydisperse bead-like chain.

\subsubsection{Confinement of crystalline}

\section{$B C P$}

Following numerous contributions on the hard confinement of semi-crystalline homopolymers including PTFE ${ }^{7}, \mathrm{PEO}^{130-133}, \mathrm{PCL}^{48,134}, \mathrm{PLLA}^{135-136}, \mathrm{PE}^{132}$, $\mathrm{PP}^{137}$, and PVDF $1-2,47,138$, researchers started investigating conventional BCPs with at least one semicrystalline block such as poly(ethylene oxide)-blockpoly( $\varepsilon$-caprolactone $) \quad(\text { PEO- } b \text {-PCL })^{139} \quad$ and poly(styrene)-block-poly(I-lactic acid) (PS-b-PLLA) $)^{140}$ under hard confinement. Confinement of conducting homopolymers and BCPs in the nanoporous AAO templates was also studied with the goal of enhancing 
piezoelectricity and conductivity type properties.

Examples of studies are on poly(3-hexylthiophene-2,5-

diyl) (P3HT $)^{141}, \quad$ poly(vinylidene-trifluoroethylene) introduce some basic concepts such as surfaceinduced crystallization and crystal orientation. ${ }^{20}$

Under hard confinement in AAO pores, both $(P(V D F-T r F E))^{142-145}, \quad$ and poly(2-methoxy-5- $\left(2^{\prime}-\quad\right.$ the crystallization $\left(T_{c}\right)$ and melting $\left(T_{m}\right)$ temperatures

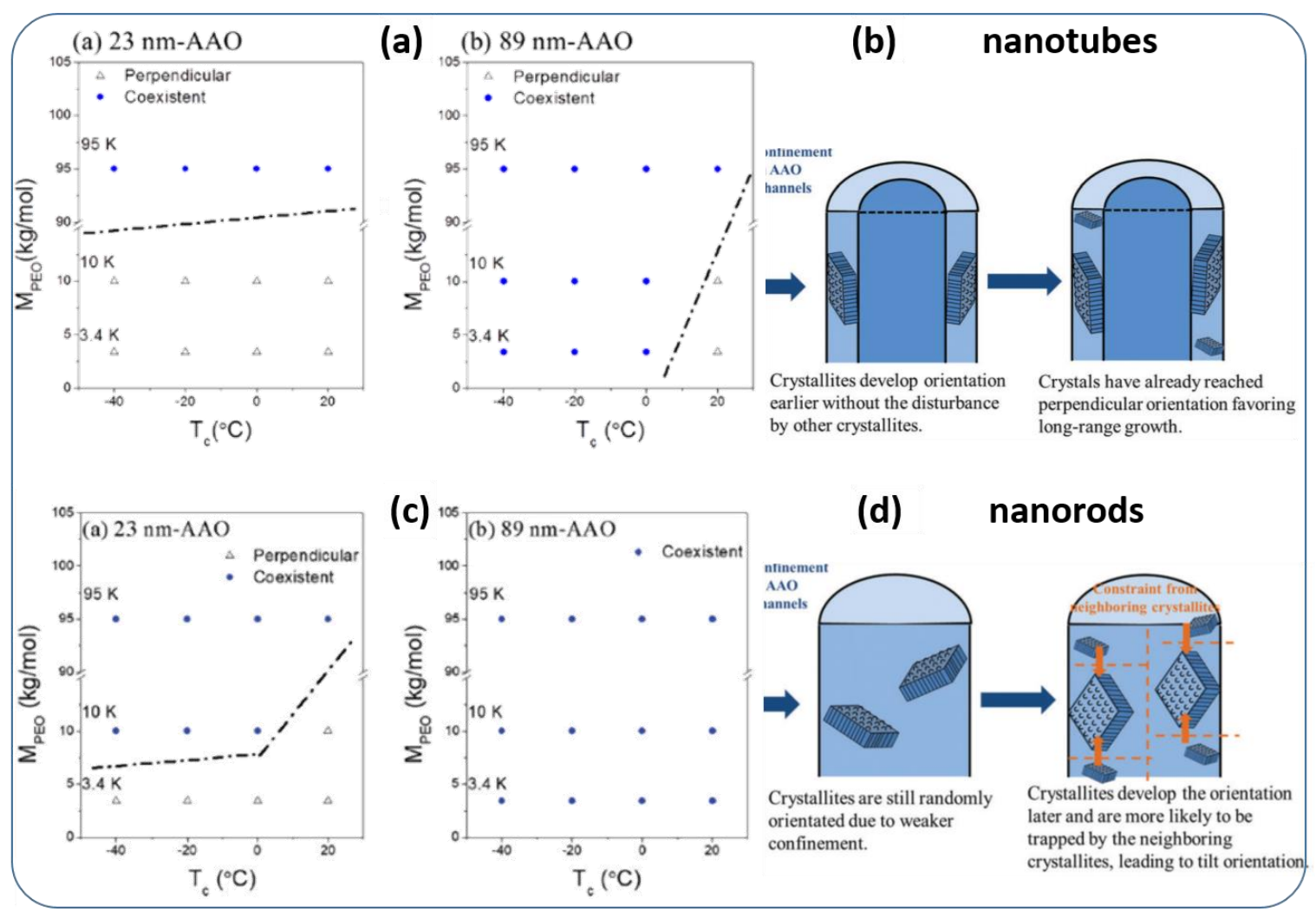

Fig. 18. Comparison of the orientation of PEO crystallites in confined nanotubes and nanorods. Orientation diagram of the crystallites in templates of 23-nm-AAO and 89-nm-AAO for the nanotubes (a) and nanorods (c), the schematic illustration of the development of the orientation of the crystallites in nanotubes (b) and nanorods (d). Reproduced from Soft Matter, 14, 5461 131 , Copyright 2018, with permission from the Royal Society of Chemistry. Reproduced from Macromolecules, 50, 631 ${ }^{149}$, Copyright 2017, with permission from the American Chemical Society.

ethylhexyloxy)-p-phenylene vinylene) (MEH-PPV). ${ }^{146}$

Numerous reviews have been totally or partially dedicated to the confinement of semi-crystalline polymers. $^{9}, 17-18,20-21,23,27-28$

First, a few precursor studies on confined semi-crystalline polymers will be summarized to of PE were reduced as the pore diameter decreased. ${ }^{147}$ The variations of $T_{c}$ are most often related to the nucleation mechanisms, from heterogeneous nucleation in bulk to surface nucleation within the nanopores. Shin et al. ${ }^{147}$ revealed that the PE chains of the lamellar crystals are perpendicular to the AAO pore axis. This was also observed for some other polymers 
including PEO ${ }^{132}$ and syndiotactic PS (sPS). ${ }^{148}$ Wu et al. showed two different orientations for SPS as a function of pore diameter. ${ }^{148}$ For smaller pores, the chains are oriented parallel to the nanopore axis (Fig. 17a).

Su et al. ${ }^{130}$ showed that in a confined state, for the PEO (10K) homopolymer probe, whatever the diameter of the AAO pores (between 40 and $400 \mathrm{~nm}$ ), a single crystallization temperature, lower than that of PEO in bulk and volume-dependent was observed. They explained these results by "clean" micro-domain nucleation (no heterogeneous nucleation). The "orientation diagram" (Fig. 17b) shows that fast cooling rates (quenching) resulted in the random distribution of PEO crystallites. A low cooling rate or large pore volume induced a mixed-orientation with $<120>$ or $<010>$ directions parallel to the pore axis (the folding plane is (120) and chain direction is the $<001>$ ). An intermediate cooling rate and small pore volume induced a crystallite orientation with a $<120>$ direction parallel to the pore axis. Under low cooling rates, growth kinetics played a decisive role in the crystal orientation and the fastest growth direction <120> tends to align parallel to the pore axis. Liu and Chen ${ }^{131}$ 149 compared the crystallite orientation of PEO homopolymers in confined nanotubes and nanorods (Fig. 18). For that purpose, they studied the crystallization behavior of PEO homopolymers of different $M_{w} s(3.4 K, 10 K$, and $95 \mathrm{~K})$ confined in $A A O$ pores (diameters of 23 and $89 \mathrm{~nm}$ ). The pores were filled using the solution infiltration method ${ }^{149}$ to obtain nanotubes and melt infiltration to generate nanorods. ${ }^{131}$ In $23 \mathrm{~nm}$ pore diameter AAO, the lowest $M_{w}$ PEO (3.4k) crystallized perpendicularly with the crystalline growing direction (stems) normal to the channel axis independent of $T_{c}$ in either nanotubes or nanorods. Increasing the $M_{w}$ of PEO or lowering the $T_{c}$ did not change the perpendicular crystal growing direction for tubular confinement, whereas it resulted in a tilt orientation for confinement in nanorods. The highest $M_{w}$ PEO (95k) showed both perpendicular and tilted orientations inside AAO templates irrespective of $T_{c}$ and type of confinement in nanotube or nanorods. In the larger pore diameter $(89 \mathrm{~nm})$, the coexistence of different orientations for nanorods was observed also independently of $M_{w}$ and $T_{c}$, whereas, for tubular confinement, low $\mathrm{M}_{\mathrm{w}}$ and high $\mathrm{T}_{\mathrm{c}}$ led to a perpendicular orientation. The narrower window of the perpendicular crystal orientation in the nanorod compared to the nanotube was due to a weaker confinement effect on the crystal growth in nanorods. The questions of nucleation, crystallization kinetics, and crystallite orientation for confined amorphoussemi-crystalline BCPs have also attracted researchers' interest. Michell et al. ${ }^{133}$ studied the crystallization of the PEO block inside PB cylinder-forming poly(ethylene oxide)-block-poly (butadiene) (PEO-bPBD) (257k) (79-21 wt\% PEO-PB) infiltrated into $35 \mathrm{~nm}$ pore diameter AAO templates by melt infiltration. In 
bulk, this diblock exhibits PB cylinders inside a matrix

of PEO domains. Consequently, the nucleation of PEO

is heterogeneous, and $T_{c}$ and $T_{m}$ are in the same ranges

as for the bulk of PEO homopolymer. Inside

nanoporous AAO templates, $T_{c}$ and $T_{m}$ of PEO of block

polymer are 72 and $15^{\circ} \mathrm{C}$ lower than that of the

corresponding bulk PEO- $b-\mathrm{PB}$, indicating that

crystallization takes place in a "heterogeneity-free

environment" where the nucleation step controls the

crystallization and crystallization kinetics quantified

with the Avrami equation ${ }^{150}$ is first-order or even

lower. ${ }^{17}$ The large supercooling-temperature gap

compared to bulk argued in favor of the change from

heterogeneous to homogeneous nucleation of PEO with a high-energy barrier required to activate

homogeneous nucleation. The question of crystallization kinetics and type of nucleation was also addressed for hard confinement of another amorphous semicrystalline BCP. ${ }^{151}$ Strongly segregated poly(ethylene)-block-poly(styrene) (PE-bPS) with a large range of PE volume fraction (11 to 79\%) with bulk morphology going from PE spheres to PS cylinders and interdomain distances between 62 and $129 \mathrm{~nm}$ were studied upon confinement inside AAO templates with a $60 \mathrm{~nm}$ pore diameter. In this case, the confinement could be considered as hard confinement because of the similarity in the characteristic distance and the pore diameter. All the

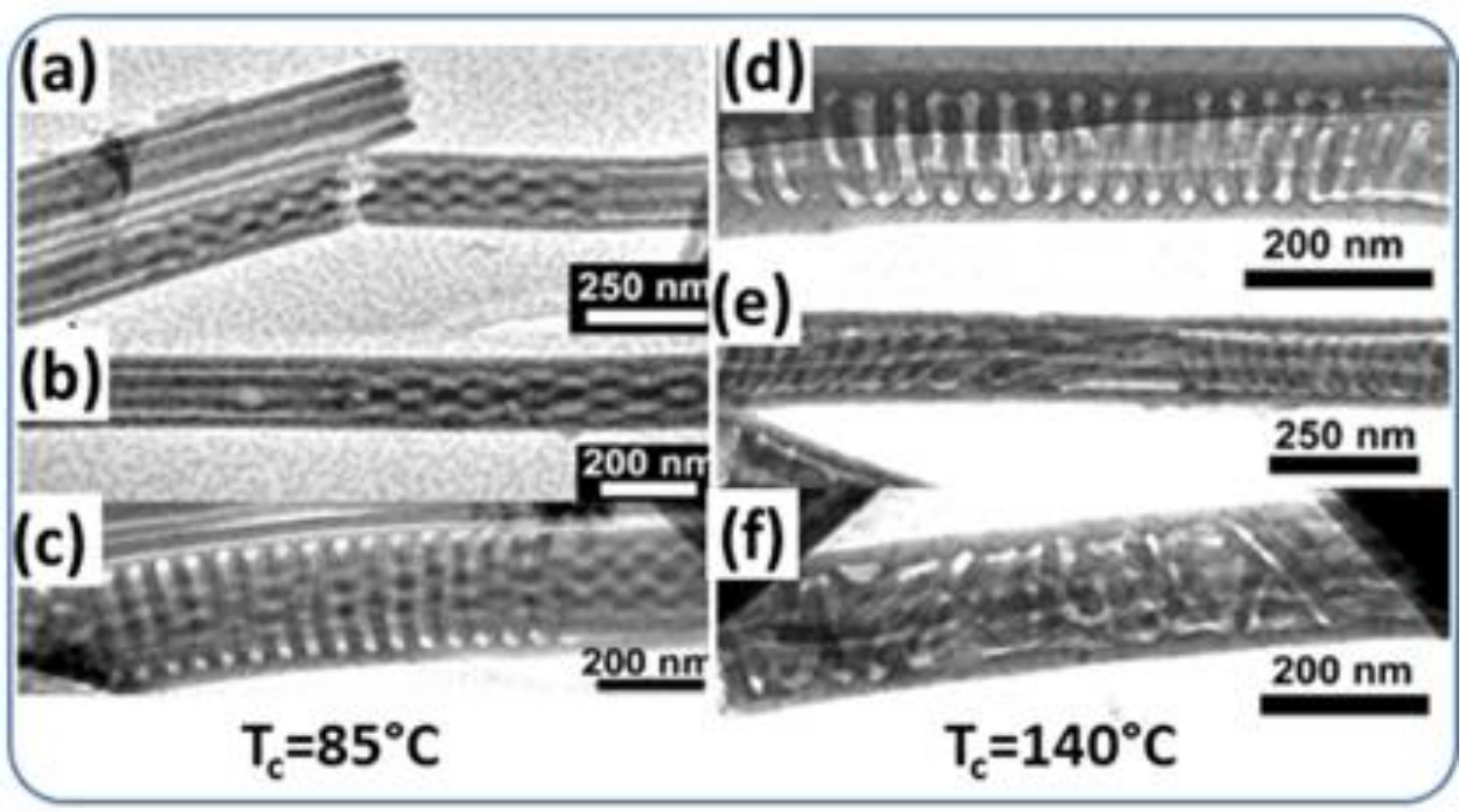

Fig. 19. TEM images of PS-PLLA nanorods obtained after isothermal crystallization at $T_{c}=85^{\circ} \mathrm{C}(\mathrm{a}),(\mathrm{b}),(\mathrm{c})$ and $\mathrm{T}_{\mathrm{c}}=$ $140^{\circ} \mathrm{C}(\mathrm{d}),(e),(f)$ for $\mathrm{D} / \mathrm{L}=4.8$ (a), (b), (e) and $\mathrm{D} / \mathrm{L}=7.3$ (c), (d), (f). Reproduced from Macromolecules, 50, 8637140, Copyright 2017, with permission from the American Chemical Society. 
shell nanorods and the tendency of PE to segregate at the AAO pore walls resulted in PE shells and PS cores.

The crystallization behavior of PE was related to surface nucleation of the PE either at the AAO pore walls or the interphase between PE and the vitreous PS block.

Yau et al. ${ }^{140}$ investigated PLLA crystallization in weekly confined $\left(D / L_{o} \geq 4\right)$ lamella-forming (PS- $b$ PLLA) (PS 21k, PLLA 24k) $\left(L_{0}=37 \mathrm{~nm}\right)$ in AAO templates with 180 and $300 \mathrm{~nm}$ pore diameters (D). Two isothermal crystallizations were studied: one at $85^{\circ} \mathrm{C}$ with PS in a glassy state and one at $140{ }^{\circ} \mathrm{C}$ with PLLA and PS still in a soft state. After isothermal crystallization at $85^{\circ} \mathrm{C}$, in addition to predominant concentric lamella along the nanopores of AAO templates, intertwined helices were observed (Fig. 19(a-c)). After isothermal crystallization at $140^{\circ} \mathrm{C}$, (Fig. 19(d-f)) more distorted helix morphologies of PS- $b$ PLLA were observed after release from AAO templates. Similar degenerated structures simultaneously present inside a rigid confining cylindrical nanopore were predicted by $\mathrm{Yu}$ et al. ${ }^{152}$ and the observed morphologies could be kinetically trapped or transient states. Crystallization at $\mathrm{T}_{\mathrm{C}}=85^{\circ} \mathrm{C}$, lower than the $\mathrm{T}_{\mathrm{g}}$ of PS, resulted in PLLAcrystallization strictly confined by the "hard" PS domains. Quenching to $T_{c}=140{ }^{\circ} \mathrm{C}$, above the $T_{g}$ of PS, PLLA crystallization is templated by the melt domain structure of the $\mathrm{BCP}$ in the middle of the nanopores. Due to the affinity of PLLA to the AAO, the outermost layer in AAO pores is PLLA and the most external cylindrical PS- $b$-PLLA shell becomes stable. In between, frustrations transferred from this most external immobilized PS- $b$-PLLA layer are accommodated by the observed morphologies. For isothermal crystallization of PS- $b$-PLLA, as of PLLA homopolymer inside AAO porosity, a preferential orientation with the faces $(110) /(200)$ normal to the nanopores' axis is observed, independently of $T_{c}$ and thus whether PS is soft or hard, contrary to nonisothermal crystallization where the (100) faces are normal to the pore axis as for heterogeneous nucleation. This hierarchical confinement by AAO nanopores and BCP melt domain structure seems to limit PLLA crystallization and heterogeneous nucleation.

The study by Suzuki et al. ${ }^{139}$ addressed the correlations between crystallization kinetics (homogeneous versus heterogeneous) and the local segmental dynamics for a confined copolymer with two crystalline blocks. Three PEO- $b$-PCL $(15,23,42 k)$ diblock copolymers with PEO (5k) fractions between 0.11 and 0.32 were confined by the melt infiltration method in AAO templates with pore diameters between 25 and $400 \mathrm{~nm}$. For PEO, the confinement is imposed by both AAO and the PCL block. It was shown that in the most asymmetric copolymer, PEO crystallization nucleates in the bulk mainly by homogeneous nucleation while within AAO templates 
PEO crystallization is completely suppressed. The crystallization of PCL blocks was also influenced by confinement and PCL crystallized at lower temperatures than in bulk via homogeneous nucleation. Using dielectric spectroscopy, the authors associated the speed-up of PCL segmental dynamics with homogeneous nucleation. They established a "phase diagram" reporting as a function of the pore curvature $(1 / d), T_{m}$ of $P C L$, heterogeneous and homogeneous crystallization temperatures using DSC and dielectric spectroscopy. Between the melt and glassy states, two intermediate metastable states corresponding to heterogeneous and homogeneous nucleation regimes at high and low temperatures were proposed. These two intermediate metastable states, which are thermodynamically less stable states than the final equilibrium state, are a classical concept in phase transformations. ${ }^{153}$

In a recent paper, Ok et al. ${ }^{154}$ showed the correlation between sequence orders and crystallization behavior of confined random THV (based on tetrafluoroethylene (TFE), hexafluoropropylene (HFP), and vinylidene fluoride (VDF)) polymer with limited alternating fractions. ${ }^{155}$ TFE monomers were found to be next to one another, while the HFP monomers, hindering the crystallization of THV because of the $\mathrm{CF}_{3}$ side groups, neighbor the VDF monomers. In THV-221G (one grade of THV with $43.8 \%$ of TFE and $46 \%$ of VDF), crystals show similarity to those of PTFE-like packing. The widening in $d$ spacing was explained by the integration of certain quantities of the HFP monomers into the THV-221G crystals. The absence of crystal orientation supports the fact that the geometric confinement has no direct effect on crystal growth in the pores. However, the $T_{m}$ evolution from ( $357 \mathrm{~K}$ ) in bulk to $338 \mathrm{~K}$ within an AAO template with a $60 \mathrm{~nm}$ pore diameter, to $332 \mathrm{~K}$ inside an AAO template with a $20 \mathrm{~nm}$ pore diameter indicates that the confinement in $A A O$ influences the crystallization of TFE chains by limiting the crystallization possibility of THV $221 \mathrm{G}$ chain segments to expand crystals. This assumption was confirmed by higher $\mathrm{T}_{\mathrm{m}} \mathrm{s}$ observed for bulk THV and confined THV415G (another grade of the terpolymer with only $0.9 \%$ of VDF) with more chain segments with crystallization possibility accessible to growing crystals, yielding larger THV 415G crystal sizes.

Almost every aspect of crystallization including crystal form, crystallinity, chain orientation, and crystallization kinetics, is affected by AAO confinement. Rather than undergoing heterogeneous nucleation as in bulk, BCPs under confinement undergo nucleation assisted by the pore surface, i.e., surface-induced nucleation. The other possibility is homogeneous nucleation that most often happens at the maximum possible super-cooling for the volume of crystallizing polymer. A general conclusion reached in 
the literature is that confinement decreases the crystallization rate for all the polymers tested so far.

\section{Dynamics of confined polymers}

\subsection{Introduction}

The mobility of polymer chains scales from local level to segmental level responsible for the liquid to glass transition, up to entire chain level. ${ }^{156}$ These motions have different time- and length- scales and are of fundamental importance since they affect the dynamic behavior of the individual chains but also the cooperative motions between chains and consequently the physical properties such as crystallization, structural and morphological properties, etc. Experimental approaches of this dynamical behavior rely mainly on NMR techniques including diffusion NMR and low-field NMR relaxometry ${ }^{157-158}$, neutron scattering ${ }^{25}$, and dielectric spectroscopies.$^{159-160}$

The spatial confinement of polymers in AAO pores affects the dynamic behavior of the individual macromolecules since the involved length for the confinement is in the same nanometer range (or tens of $\mathrm{nm}$ ) as for the dynamic behavior. When considering the studies on dynamics of confined polymers, it is important to note that most of the dynamical studies concern single homopolymer, very few papers are dealing with block copolymers and none with polymers blends. Since some excellent reviews focused on the polymer dynamics under confinement
$18,21,25$ it is out of the scope of this paper to present an exhaustive review of the literature. The main features of the dynamics of homopolymers in AAO rigid confinement are shortly recalled in this paper through the new published papers since these reviews. The few existing works on BCP will be covered in more details.

\subsection{Confined homopolymers}

Dynamics of homopolymers were studied for numerous homopolymers, either entangled or unentangled polymers. Typical studied polymers were polyisoprene $(\mathrm{PI})^{159-161}$, poly(1,4-butadiene) $(\mathrm{PBD})^{162-}$ 165 polyethylene oxide (PEO) ${ }^{166}$, PVDF$^{138}$, PDLLA $^{135}$, $\mathrm{PCL}^{48}, \quad$ PPG ${ }^{167-168}$, poly(methylphenylsiloxane) ${ }^{169}$, poly(dimethylsiloxane) ${ }^{170}$, polyethylene-alt-propylene (PEP). ${ }^{171}$ Alexandris et al. studied the dynamical behavior of unentangled cis-1,4-polyisoprene (PI) ( $\mathrm{M}_{\mathrm{w}}$ $=300$ to 5000) inside AAO with pore diameter from 25 to $400 \mathrm{~nm}$ using dielectric spectroscopy. ${ }^{159}$ The cooperative segmental mode and the chain mode were observed at the same position as for the bulk polymer with, however, a pronounced enlargement of relaxation times even inside pores with dimension 50 times the unperturbed chain sizes. These observations are explained by chains adsorption on AAO pore walls and global chain relaxation retarded by the confinement. The glass transition temperature was unaffected by the confinement. Politidis et al. examined the confinement of the entangled cis-1,4polyisoprene $\mathrm{PI}\left(\mathrm{Mw}\right.$ between $8.5 \mathrm{k}$ and $100 \mathrm{k}$ ). ${ }^{160}$ 
Under confinement, as for unentangled chains, the $\alpha$ relaxation (segmental mode) and $\beta$-relaxation (chain mode) processes became broader but a new relaxation process appeared for entangled chains between these two last modes. Named intermediate process, it became dominant as the confinement degree increased and was attributed to adsorption/desorption processes of the polymer segments on the pore wall. Moreover, the liquid-toglass transition temperature changed under confinement degree unlike untangled PI chains. This last result confirms the relationship between the glass transition temperature of the polymer chains under confinement and the interfacial energy of between polymer and pore wall proposed by Alexandris et al. ${ }^{172}$ The higher the interfacial energy is, the higher Adrjanowicz et al. ${ }^{169}$ studied the dynamical behavior of poly(methylphenylsiloxane) $\left(M_{w}=21 k\right)$ inside $A A O$ pores with diameter ranging from 10 to $120 \mathrm{~nm}$ and its pressure dependence. Three liquid-to-glass transitions were observed, respectively attributed to the molecules at the interface with the pore wall, the core of pores and the interlayer between these two regions. Shi et al. ${ }^{135}$ studied the segmental dynamics of PDLLA $\left(\mathrm{M}_{\mathrm{w}}=20 \mathrm{k}\right)$ in nanoporous alumina (pore diameter between 40 and $400 \mathrm{~nm}$ ). Two segmental relaxation loss peaks corresponding to two glass transition temperatures were recorded, one is the same as for the bulk, the other lower and related to higher segmental mobility at the AAO surface. By applying well-chosen protocols for the measurements, these authors highlighted an important dependence of the segmental dynamics in AAO pores with the thermal history of the polymer and the importance of the non-equilibrium nature of chain segments under confinement and of the kinetic to reach equilibrium. The enhanced segmental mobility at the AAO surface could explain the crystallization behavior of PLLA under confinement with a shift of crystallization temperature toward higher temperature. 


\subsection{Confined block copolymers}

Dynamical studies on BCPs in confined environments in AAO are still scarce. ${ }^{139,173-174}$ Dynamics of PS cylinder forming PS-b-PI diblock copolymer was explored under block, the segmental relaxation mode of isoprene block, and the chain relaxation mode of isoprene block

(Fig. 20(a)). The dynamic $T_{g}$, arising from segmental relaxation motions of the styrene and isoprene blocks 2D nanoconfinement in AAO pores (pore diameter 18,

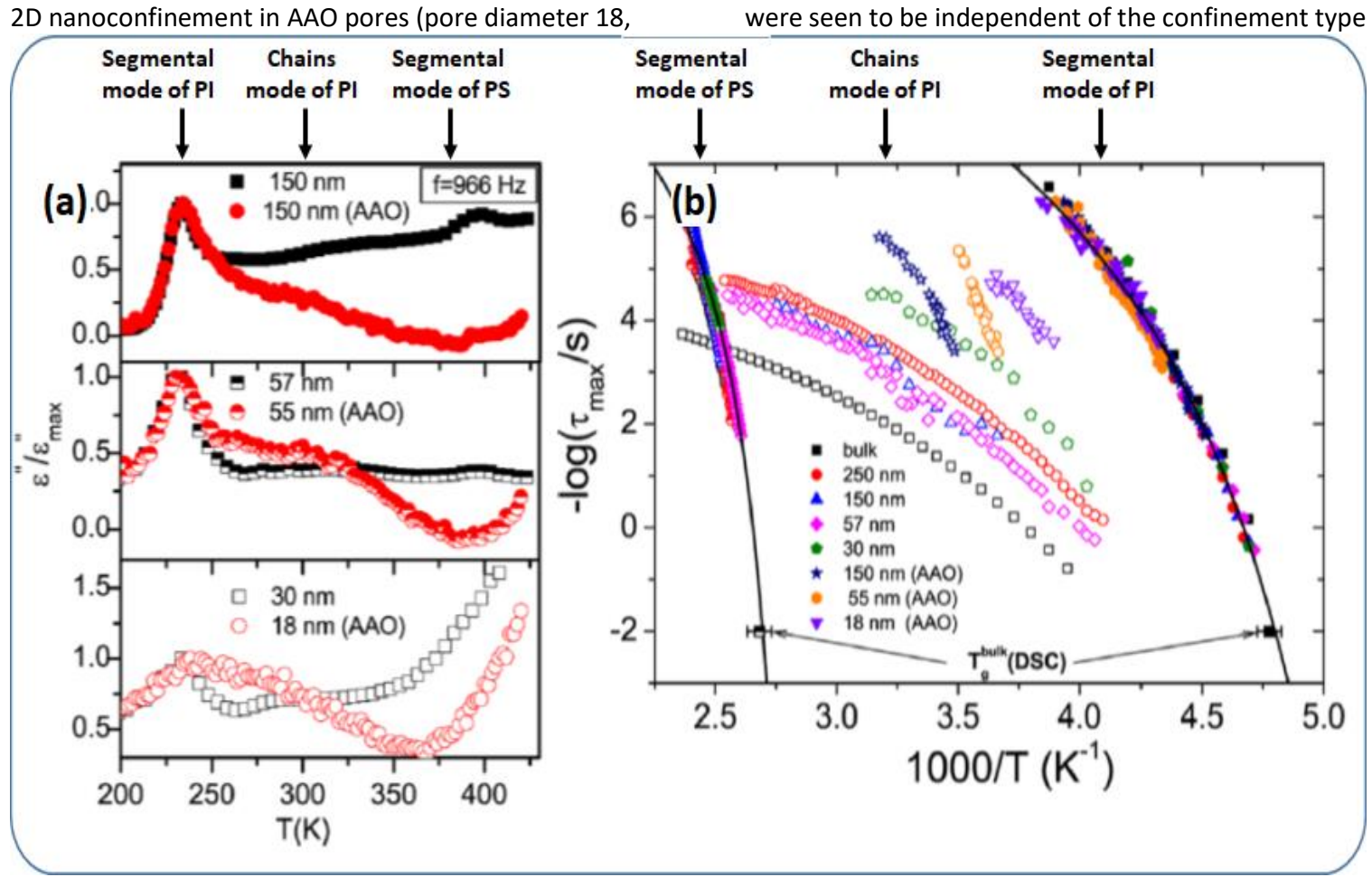

Fig. 20. Dynamic of PS-b-PI in thin films and in AAO nanopores. Isochronal $(966 \mathrm{~Hz})$ dielectric loss spectra obtained in thin films (black squares) and in AAO nanopores (red circles) for different film thickness and AAO pore sizes (a), Activation plot of the relaxation rates corresponding to the segmental mode of PI (filled symbols), normal mode of PI (open symbols), and segmental mode of PS styrene blocks (half-filled symbols) for PS-b-PI the bulk state, and when confined in thin films and in AAO nanopores as indicated in the legend. Bulk PI and PS homopolymers $\mathrm{T}_{\mathrm{g}}$ from DSC. Reproduced from ACS Appl. Mater. Interfaces, 2015, 7, 12328 173 , Copyright 2015, with permission from the American Chemical Society.

$55,150 \mathrm{~nm}$ ) and compared to 1D nanoconfinement in (1D or 2D) (Fig. 20(a)) and size (independent of the film thin films with the same thicknesses as pore thickness and pore diameter (Fig. 20(b)). However, the diameters. ${ }^{175}$ Broadband dielectric spectroscopy spectra exhibited three dielectrically active relaxation confinement type and size affect the chain dynamics due to change of morphology with the confinement

signals: the segmental relaxation mode of the styrene 
size and chain adsorption and interaction on pore wall or substrate (Fig. 20 (b)).

Maiz et al. ${ }^{174}$ investigated the dynamics of P4VP in P4VP cylinder-generating PS-b-P4VP in bulk and under confinement in nanoporous AAO templates with 25, 35, 60 and $300 \mathrm{~nm}$ pore diameters. They found that the segmental dynamics of P4VP within the largest pores $(65,330 \mathrm{~nm})$ was slower than that of the bulk BCP but turned out to be more rapid in smaller pores $(35,25 \mathrm{~nm})$. The presence of gold nanoparticles in P4VP domains is shown to enhance this effect.

In the case of semi-crystalline PEO-b-PCL diblock copolymers confined into nanoporous AAO templates with pore diameters ranging from 25 to 400 $\mathrm{nm}$, the kinetics of crystallization is shown to be related it to the segmental dynamics. ${ }^{139}$ The major $\mathrm{PCL}$ block crystallizes at lower temperatures via homogeneous nucleation in the confined geometry. This nucleation, as revealed by dielectric spectroscopy, was governed by an accelerated segmental dynamics in the confined geometry. A similar acceleration in the segmental dynamics was observed for hyperbranched PEO confined into AAO templates. In this case, this leads to the suppression of the crystallization. ${ }^{96}$

To summarize, a great deal of work has shown that dynamical behaviors of confined homopolymers inside AAO templates can be deeply modified due to pronounced polymer/wall interaction. Future work should be now directed to dynamics of
BCPs and polymers blends inside AAO templates, and their relations to properties such as phase separation and self-assembly, which are still uncharted territories.

\section{Conclusion and future trends}

Polymers under confinement represent a major scientific topic because confinement modifies their characteristics, opening a vast land of opportunity to tune their properties. In this review, we focused on the changes of the phase properties (nanostructure and morphology) and crystallization behavior of inhomogeneous polymers such as polymer-polymer blends or block copolymers (BCP) under two-dimensional (2D) confinement in anodic aluminum oxide (AAO) templates. We summarize below the most striking features of this in-depth analysis of the signs of progress made during the last decade and also provide directions for future work.

\subsection{Block copolymer area}

In a very good review summarizing the research published over the first decade of this century, Shi et al. ${ }^{16}$ recognize that the behavior of simple BCP in a confined environment was already well deciphered and they emphasized in their concluding remarks that confining $\mathrm{BCP}$ with architectures that are more complex, or multicomponent systems, may open a new land of morphologies. Almost a decade later, we have shown in this review that researchers have followed this trend, searching for more complexity in the obtained morphologies. Below are the new 
concepts and/or principles beyond what we have learned prior to 2010 that have been implemented in this quest for complexity.

\subsubsection{New processing methods}

The "double solution wetting method" in which templates are dipped in two different solutions in sequential order, constituted in our opinion one important achievement of the considered period. It allows complex morphologies to be formed starting from simple system like diblock, to produce a greater diversity of nanostructures such as nanospheres, and porous nanorods. Only implemented for a limited number of systems, this approach appears to us as a promising tool to produce a myriad of original complex morphologies, by selecting the new combination of polymer, solvent and surface chemistry of the pore.

\subsubsection{Post-treatments}

Annealing approaches such as thermal annealing, SAINT, and SVA opened new routes for governing polymers under confinement inside AAO nanopores.

In comparison to the vast amount of work devoted to SVA in the thin film situation, this area is still in its infancy and certainly deserves more investigations. Even if the phase diagram in confinement has been well described through simulation, unpredicted morphologies can be observed experimentally in this case since the simulation predicts the most thermodynamically stable morphology whereas a metastable morphology (resulting from kinetic effects) can also be formed experimentally. The dried morphology indeed results from a complex interplay of the phenomena occurring during swelling and deswelling. This opens new perspectives, in which the number of parameters to be controlled is high, but it is very promising for the generation of new and original morphologies. In this regard, the SAINT method appears as a very appealing process since it combines infiltration and phase behavior control, due to the presence of solvent vapor throughout the whole process.

Another major tool to tune behaviors of confined polymers is thermal annealing. As shown by Shingne et al. ${ }^{2}$, elevated crystallization temperatures along with lengthy annealing durations helped with the modification of PVDF, and generated $\gamma$ domains. Thermal treatment was also utilized to suppress the crystallization of PEO block in PEO- $b$-PCL confined into AAO nanopores. ${ }^{139}$

\subsubsection{Selective removal}

One important achievement in the last decade has been the selective removal of one block of a confined $B C P$ inside $A A O$, opening space for infiltrating new functional materials such as inorganic nanoparticles. Hence, the confined BCP could also function as a soft template under hard confinement: one phase of the BCP might be selectively loaded with inorganic nanoparticles with certain functionalities so that, for example, nano-capacitors or nano-cables could be generated. We only recorded a limited 
amount of literature on such applications, which certainly needs more investigations in order to get the full potential of the method.

\subsubsection{Unconventional $A A O$}

In the second half of the last decade, new signs of progress were also observed in the design of unconventional anodic aluminum oxide (AAO) templates, opening new opportunities to prepare polymeric nanostructures with more complex shapes (conical, branched, etc.). The literature dealing with filling such advanced templates is still scarce ${ }^{31,42}$, and certainly deserves more attention from the polymer community. Surface functionalization of AAO pore walls can also influence structural changes in BCP blends inside AAO templates. Reusable replica templates obtained using AAO membranes developed by Zhang et al. ${ }^{50}$ could be used to study the properties of confined novel BCPs. As stated by Lee and Park ${ }^{36}$, over the past approximately 100 years, several electrochemical aspects of aluminum anodization have been well established. During the last two decades, there is an enormous increase in applications of AAO templates as templates for various purposes including generation of polymeric nanotubes and nanowires. Proper engineering of characteristics of AAO templates such as pore diameter, depth, and surface functionalities by new approaches will further expand the applications. Therefore, the future prospects of AAO templates for nanotechnology applications of polymers are promising.

\subsubsection{Homopolymer/BCP blends}

One of the great advances made in the last decade concerns the use of homopolymer/BCP blends, leading to more complex morphologies. It can also be anticipated that novel BCPs, resulting from advancements in the area of polymer synthesis, could be investigated under hard confinement inside $A A O$ templates to generate even more complex organizations. For example, it has been predicted by simulation that star polymers (that would normally form Archimedean patterns in the bulk) will adopt a rich set of morphologies ${ }^{175}$ but this has not been experimentally confirmed yet. Generally speaking, we found that research data concerning triblock copolymers was still scarce and certainly represent a major goal for future trends. Studies on the confinement of $B C P / B C P$ blends in thin films at solid inter-phases could also be extended by focusing on $\mathrm{BCP} / \mathrm{BCP}$ blends under hard confinement.

\subsubsection{Dynamics of Confined Polymers}

Local segmental dynamics of confined polymers might affect different properties such as crystallization. An important lesson learned on dynamics of confined polymers is that confining influences $T_{g}, T_{m}$, and $T_{c}$ of confined polymers. Based on the lessons learned about the changes in $T_{g}$ and $T_{c}$ of confined polymers, we suggest that further work is needed on confined BCPs to reveal both dynamics and deviations in $T_{m}, T_{c}$, and $T_{g}$ of different blocks of BCPs. The changes in these temperature values could be 
utilized to tune different properties such as suppression of crystallization of BCPs.

\subsubsection{Simulations}

Progress in computational capabilities could enable simulations within a short time. This might in turn be beneficial in designing complicated experiments to investigate, for example, BCP blends under confinement inside hard templates with different pore geometries such as conical ones. Hence, theoretical work and simulations based on an experimental database have to be complementary and should provide insight into better visualization and understanding of confined BCPs. Theoretical contributions, as discussed by Ha et al. ${ }^{113}$, on confined novel BCPs could also reflect information on "real-life" problems as well as concepts/processes to be discovered/described in natural systems. Simulations to be performed on novel BCPs confined in new generation AAO templates with different pore shapes including funnels and pencils open a new area for computational and theoretical researchers.

\subsection{Polymer blends area}

In comparison to the BCP field that benefited from a great deal of work in the first decade of this century, studies concerning polymer blends only really emerged within the last decade. Introducing polymer blends into such a confined environment leads to heterogeneities at the nanoscale, resulting from complex phenomena such as wetting/dewetting, capillarity, phase separation, flow instabilities, vitrification, etc. Depending on the mode of infiltration (solvent wetting or melt infiltration), the relative importance of these parameters varies. A given polymer blend composition can thus result in different types of morphologies, driven by the infiltration process. In comparison to the BCP case, the infiltration of polymer blends exhibits a distinct behavior in melt infiltration. Since the homopolymers in a polymer blend can behave independently (in contrast to the BCP case where the blocks are covalently linked), the differential viscous flow of the components can result in very specific heterogeneous structures inside the pores. Gradients of homopolymer concentration can be generated through the axis of the pore, a very specific behavior that cannot be attained with BCPs. Sequential infiltration of homopolymers into nanoporous AAO templates could be employed in a clever way to form Janus rods. In summary, and because polymer blends in confinement behave very specifically compare to block-copolymer, this area needs more specific investigations.

One of the current research challenges in polymer science is the understanding of the fundamentals of polymer crystallization. Even in bulk, a huge number of questions still remain on the nucleation mechanism, on the transformation of initially flexible polymer chains into rigid ordered segments and amorphous segments, the crystal growth mechanism, the melt memory effects. Unified 
models are still lacking. ${ }^{176-177}$ Compared to bulk, understanding the fundamentals of polymer crystallization under confinement is an even greater challenge, with questions about the influence of confinement strength, interfacial energy between pore walls and polymers and the adsorption layer on the crystallization mechanisms. It is expected that more theoretical and experimental achievements will be made to answer the remaining questions and to overcome the challenges in the coming decade. Answering these questions might contribute to a better understanding of the development of polymer crystallization theory and potential applications of crystalline polymers under confinement. Tuning the crystallization of polymers in confined geometry could be used to tailor the confined polymer crystallization properties for nanotechnology and many other novel applications. ${ }^{176}$

In conclusion of this review, we believe that despite the considerable amount of work already devoted to this topic in the $2000-2020$ period, there is plenty of room at the bottom in the nano-world for confined polymers and BCPs. Particularly, experimental work still requires further exploration and investigations in the coming decade, to fill the gap with theoretical studies.

\section{Author Contributions}

Each of the three authors is equal contributor to writing and editing of the current manuscript.

\section{Conflict of interest}

The authors declare no conflict of interest.

\section{Acknowledgments}

S.O. is thankful to Dr. Dawood Bahzad, Science and Technology Director of Petroleum Research Center at Kuwait Institute for Scientific Research, for his encouragement at a very early stage of this review article.

\section{References}

1. C. Fu, X. Wang, X. Shi, and X. Ran, RSC Adv., 2015, 5, 87429-87436.

2. N. Shingne, M. Geuss, T. Thurn-Albrecht, H.-W. Schmidt, C. Mijangos, M. Steinhart, and J. Martin, J. Phys. Chem. B, 2017, 121, 7723-7728.

3. C.R. Martin, Adv. Mater., 1991, 3, 457-459.

4. C.R. Martin, Science, 1994, 266, 1961-1966.

5. C.R. Martin, Acc. Chem. Res. 1995, 28, 61-68.

6. C.R Martin, Chem. Mater., 1996, 8, 1739-1746.

7. M. Steinhart, J. H. Wendorff, A. Greiner, R. B. Wehrspohn, K. Nielsch, J. Schilling, J. Choi, and U. Gösele, Science, 2002, 296, 1997.

8. G Song, X. She, Z. Fu, and J. Li, J. Mater. Res., 2004, 19, 3324-3328.

9. M. Steinhart, Adv. Polym. Sci., 2008, 220, 123-187. 10. Y. Wang, Y. Qin, A. Berger, E. Yau, C. He, L. Zhang, U. Gösele, M. Knez, and M. Steinhart, Adv. Mater., 2009, 21, 2763-2766. 
11. M. Ma, E. L. Thomas, G. C. Rutledge, B. Yu, B. Li, Q. Jin, D. Ding, and A.-C. Shi, Macromolecules, 2010, 43, $3061-3071$

12. H. Xiang, K. Shin, T. Kim, S.I. Moon, T.J. McCarthy, and T.P. Russell, Macromolecules, 2004, 37, 56605664.

13. P. Dobriyal, H. Xiang, M. Kazuyuki, J. Chen, H. Jinnai, and T.P. Russell, Macromolecules, 2009, 42, 9082-9088.

14. Y.M. Sun, M. Steinhart, D. Zschech, R. Adhikari, G.H. Michler, and U. Gösele, Macromol. Rapid Comm., 2005, 26, 369-375.

15. C.R. Stewart-Sloan, and E.L. Thomas, Eur. Polym. J., $2011,47,630-646$.

16. A.-C. Shi, and B. Li, Soft Matter, 2013, 9, 13981413.

17. R.M. Michell, I. Blaszcyzyk-Lezak, C. Mijangos, and A.J. Müller, J. Polym. Sci. Part B: Polym. Phys., 2014, 52, 1179-1194.

18. C. Mijangos, R. Hernandez, and J. Martin, Prog. Polym. Sci., 2016, 54-55, 148-182.

19. P. Huber, J. Phys.: Condens. Matter, 2015, 27, 103102-1-103102-43.

20. R.M. Michell, and A.J. Muller, Prog. Polym. Sci., 2016, 54-55, 183-213.

21. H. Wu, Y. Higaki, and A. Takahara, Prog. Polm. Sci., 2018, 77, 95-117.

22. Q. Xu, G. Meng, and F. Han, Prog. Mater. Sci., 2018, 95, 243-285.
23. P. Samanta, C.-L. Liu, B. Nandan, and H.-L. Chen, Multiphase polymer systems, eds. S. Thomas, A.P. Mohammed, E.B. Gowd, and N. Kalarikkal, Elsevier, Amsterdam, 2018, Chapter 13, 367-431.

24. C.-W. Chang, H.-W. Ko, and J.-T. Chen, Nano/microstructured materials for energy and biomedical applications, eds. B. Li, and T. Jiao, Springer, Singapore, 2018, Chapter 5, 165-203.

25. D. Richter, and M. Kruteva, Soft Matter, 2019, 15, $7316-7349$.

26. A. Rath, and P. Theato, Adv. Funct. Mater., 2020, 30, 1902959-1-1902959-16.

27. M.-C. Lin, B. Nandan, and H.-L. Chen, Soft Matter, $2012,8,7306-7322$.

28. S. Nakagawa, H. Marubayashi, and S. Nojima, Euro. Polym. J. 2015, 70, 262-275.

29. C.J. Ingham, J. ter Maat, and W.M. de Vos, Biotechnol. Adv. 2012, 30, 1089-1099.

30. H. Buff, Liebigs Ann. Chem. 1857, 3, 265-284.

31. 152. W. Lee, and S-J. Park, Chem. Rev., 2014, 114, 7487-7556.

32. H. Masuda, and K. Fukuda, Science, 1995, 268, 1466-1468.

33. A. Ruiz-Clavijo, O. Caballero-Calero, and M. MartinGonzalez, Nanoscale, 2021, 13, 2227-2265.

34. L. Li, J. Liu, L. Qin, C. Zhang, Y. Sha, J. Jiang, X. Wang, W. Chen, G. Xue, and D. Zhou, Polymer, 2017, 110, $273-283$. 
35. H. Wu, Z. Su, and A. Takahara, Soft Matter, 2011, 7, 1868-1873.

36. Z. Zhou, and S.S. Nonnennmann, Materials, 2019, 12, 2535-1-2535-19.

37. A. P. Li , F. Muller, A. Birner, K. Nielsch, and U. Gösele, J. Appl. Phys., 1998, 84, 6023-6026.

38. H. Masuda, F. Hasegwa, and S. Ono, J. Electrochem. Soc., 1997, 144, L127-L130.

39. A.M. Md. Jani, D. Losic, and N.H. Voelcker, Prog. Mater. Sci., 2013, 58, 636-704.

40. C. Li, J. Li, C. Chen, and J. Zhu, Chem. Comm., 2012, $48,5100-5102$.

41. J. Li, C. Li, C. Chen, Q. Hao, Z. Wang, J. Zhu, and X. Gao, ACS Appl. Mater. Interfaces, 2012, 4, 5678-5683. 42. Y. Kim, A. Kumagai, X. Hu, A.-C. Shi, B. Li, H. Jinnai, and K. Char, Macromolecules 2019, 52, 4803-4811.

43. M. Raoufi, and H. Schönherr, RSC Adv. 2013, 3, 13429-13436.

44. A.Y.Y. Ho, H. Gao, Y.C. Lam, and I. Rodriguez, Adv. Func. Mater. 2008, 18, 2057-2063.

45. S. Liu, S. Tang, H. Zhou, C. Fu, Z. Huang, H. Liu, and Y. Kuang, J. Solid State Electrochem., 2013, 17, 19311938.

46. L. Wen, R. Xu, Y. Mi, and Y. Lei, Nature Nanotech., 2017, 12, 244-252.

47. X. Dai, J. Niu, Z. Ren, X. Sun, and S. Yan, J. Phys. Chem. B, 2016, 120, 843-850.

48. Y. Suzuki, H. Duran, W. Akram, M. Steinhart, G. Floudas, and H.-J. Butt, Soft Matter, 2013, 9, 91899198.
49. C.-T. Liu, Y.-L. Lin, C.-W. Chu, C.-W. Chang, Y.-J. Chiu, T.-Y. Chiu, L.-R. Lee, and J.-T. Chen, J. Phys. Chem. C, 2019, 123, 14540-14546.

50. J. Zhang, S. Shen, X.X. Dong, and L.S. Chen., Optics Exp. 2014, 22, 1842-1851.

51. J.-T. Chen, C.-W. Lee, M.-H. Chi, and I-C. Yao. Macromol. Rapid Commun. 2013, 34, 348-354.

52. M. Steinhart, P. Goring, H. Dernaika, M. Prabhukaran, U. Gösele, E. Hempel, and T. ThurnAlbrecht, Phys. Rev. Lett., 2006, 97, 027801-1-0278014.

53. M.F. Zhang, P. Dobriyal, J.T. Chen, T.P. Russel, J. Olmo, and A. Merry, Nano Lett., 2006, 6, 1075-1079. 54. O. Kriha, L.L. Zhao, E. Pippel, U. Gösele, R.B. Wehrspohn, J.H. Wendorff, M. Steinhart, and A. Greiner, Adv. Functional Mater., 2007, 17, 1327-1332. 55. S. Jin, Y. Lee, S.M. Jeon, B.H. Sohn, W.S. Chase, and J.K. Lee, J. Mater. Chem., 2012, 22, 23368-23373.

56. J.-T. Chen, T.-H. Wei, C.W. Chang, H.-W. Ko, C.-W. Chu, and M.-H. Chi, Macromolecules 2014, 47, 52275235.

57. C.W. Chang, M.H. Chi, C.W. Chu, H.W. Ko, Y.H. Tu, C.C. Tsai, and J.-T. Chen, RSC Adv., 2015, 5, 2744327448.

58. S.F. Ai, Y. Cui, Q. He, C. Tao, and J.B. Li, Coll. Sur. A, $2006,275,218-220$.

59. J. Martin, J. Maiz, J. Sacristan, and C. Mijangos, Polymer 2012, 53, 1149-1166. 
60. T.-C. Wang, H.-Y. Hsueh, and R.-M. Ho, Chem. Mater., 2010, 22, 4642-4651.

61. C.-J. Chu, M.-H. Cheng, P.-Y. Chung, M.-H. Chi, K.-S. Jeng, and J.-T. Chen., Int. J. Polym. Mater. Polym. Biomater., 2016, 65, 695-701.

62. I-C. Yao, M.-H. Cheng, and J.-T. Chen, Macromol. Chem. Phys. 2015, 216, 2154-2160.

63. H.-W. Ko, M.-H. Chi, C.-W. Chang, C.-W. Chu, K.-H. Luo, and J.-T. Chen, ACS Macro Lett., 2015, 4, 717-720.

64. S. Mei, X. Feng, and Z. Jin, Soft Matter, 2013, 9 945951.

65. L. Brock, and J. Sheng, Micromachines, 2020, 11, 46-1-46-20.

66. V.M. Cepak, and C. R. Martin, Chem. Mater., 1999,

11, 1363-1367.

67. C.-W. Chang, M.-H. Cheng, H.-W. Ko, C.-W. Chu, Y.-

H. Tu, and J.-T Chen, Soft Matter, 2018, 14, 35-41.

68. D. Borah, S. Rasappa, R. Senthamaraikannan, J.D.

Holmes, and M.A. Morris, Polymers, 2015, 7, 592-609.

69. C. Jin, J.N. Murphy, K.D. Harris, and J.M. Buriak, ACS Nano, 2014, 8, 3979-3991.

70. S. Ok, J. Sheets, S. Welch, S. Kaya, A. Jalilov, and D.R. Cole, J. Polym. Sci., Part B: Polym. Phys., 2017, 55, $643-657$.

71. C.W. Lee, T.H. Wei, C.W. Chang, and J.T. Chen, Macromol. Rapid Commun., 2012, 33, 1381-1387.

72. M.H. Chi, Y.H. Kao, T.H. Wei, C.W. Lee, and J.T.

Chen, Nanoscale 2014, 6, 1340-1346.
73. Y.C. Huang, P.W. Fan, C.W. Lee, C.W. Chu, C.C. Tsai, and J.T. Chen, ACS Appl. Mater. Interfaces, 2013, 5, 3134-3142.

74. P. Muanchan, S. Suzuki, T. Kyotani, and H. Ito. Polym. Eng. Sci., 2017, 57, 214-223.

75. L. Xue, J. Zhang, and Y. Han, Prog. Polym. Sci., 2012, 37, 564-594.

76. A.R. Ajitha, and S. Thomas, In: A.R. Ajitha, and S. Thomas, (Eds.) Compatibilization of polymer blends micro and nano scale phase morphologies, interphase characterization and properties, Elsevier, Amsterdam, 2020, Chapter 1, 1-29.

77. E.L. Thomas, and R.L. Lescanec, Phil. Trans. R. Soc. Lond. A., 1994, 348, 149-166.

78. S.Y. Heriot, and R. A. L. Jones, Nat. Mater., 2005, 4, 782-786.

79. Y. Mouhamad, P. Mokarian-Tabari, R.A.L. Jones, and M. Geoghegan, Polymer 2019, 178, 121578-1121578-3.

80. J. Rodriguez-Hernandez, Recent developments in polymer macro, micro and nano blends, eds. P.M. Visakh, G. Markovic, and D. Pasquini, Woodhead Publishing, Cambridge, 2017, Chapter 6, 131-161.

81. A.J. Muller, V. Balsamo, and M.L. Arnal, Adv. Polym. Sci., 2005, 190, 1-63.

82. A. Vital, M. Vayer, T. Tillocher, R. Dussart, M. Boufnichel, and C. Sinturel, Appl. Surface Sci., 2017, 393, 127-133. 
83. C. Sinturel, M. Vayer, M. Morris, and M.A. Hillmyer, Macromolecules, 2013, 46, 5399-5415.

84. H.-J. Chung, H. Wang, J. Russell, and A. Composto, Macromolecules, 2006, 39, 153-161.

85. D.U. Ahn, Z. Wang, I.P. Campbell, M. P. Stoykovich, and Y. Ding, Polymer, 2012, 53, 4187-4194.

86. D. Chen, J.-T. Chen, E. Glogowski, T. Emrick, and T.P. Russell, Macromol. Rapid Commun., 2009, 30, 377-383.

87. B.S. Gupta, and A.K. Moghe, Biotextiles in medical implants, ed. F. Dodds, Woodhead Publishing, Oxford, 2013, Chapter 2, 48-90.

88. L. Rayleigh, Proc. London Math. Soc., 1878, 10, 413.

89. T.-H. Lee, Y.-J. Chiu, Y.-C. Lai, P.-W. Fan, T.-Y. Kuo, I. Liau, and J.-T. Chen, RSC Adv., 2014, 4, 51884-51892. 90. P. Muanchan, T. Kurosei, and H. Ito, Polymers 2019, 11, 1039-1-1039-18.

91. H.-W. Ko, M.-H. Cheng, M.-H. Chi, C.-W. Chang, and J.-T. Chen, Langmuir, 2016, 32, 2110-2116.

92. W. Tian, L. Huang, D. Zang, C. Li, J. Danga, and D. Wang, RSC Adv., 2014, 4, 53021-53027.

93. H. Wu, Z. Su, and A. Takahara, Polymer Journal, 2011, 43, 600-605.

94. H. Wu, Z. Su, Y. Terayama, and A.Takahara, Sci. Chi.

Chem., 2012, 55, 726-734.

95. H. Wu, Z. Su, and A.Takahara, RSC Adv., 2012, 2, 8707-8712.
96. Y. Yao, Y. Suzuki, J. Seiwert, M. Steinhart, H. Frey, H.-J. Butt, and G. Floudas, Macromolecules, 2017, 50, 8755-8764.

97. Y. Yao, H.-J. Butt, J. Zhou, M. Doi, and G. Floudas, Macromolecules, 2018, 51, 3059-3065.

98. S. Dougherty, and J. Liang, Nanotechnology, 2009,

20, 295301-1-295301-7.

99. B. Sanz, I. Blaszczyk-Lezak, C. Mijangos, J.K. Palacios, and A.J. Muller, Langmuir, 2016, 32, 78607865.

100. H.-W. Ko, M.-H. Chi, C.-W. Chang, C.-W. Chu, K.H. Luo, and J.-T. Chen, ACS Macro Lett., 2015, 4, 717720.

101. H. Feng, X. Lu, W. Wang, N.-G. Kang, and J.W. Mays, Polymers, 2017, 9, 494-524.

102. A-C. Shi, and B. Li, In: K. Matyjaszewski, and M. Moller (Eds.), Polymer science: a comprehensive reference, Newnes, Oxford, 2012, Volume 7, 71-81.

103. L. Leibler, Macromolecules, 1980, 13, 1602-1617. 104. F.S. Bates, and G.H. Fredrickson, Annu. Rev. Phys. Chem., 1990, 41, 525-557.

105. E. Helfand, and Z.R. Wasserman, Developments in block copolymers 1, ed. I. Goodman, Elsevier, New York, 1982, Chapter 4, 99-125.

106. G.H. Fredrickson, and E. Helfand, J. Chem. Phys., $1987,87,697-705$.

107. D.H. Kim, and S.Y. Kim, J. Phys. Chem. Lett., 2017,

8, 1865-1871. 
108. W. Li, M. Liu, and F. Qiu, J. Phys. Chem. B, 2013, $117,5280-5288$.

109. T. P. Russell, and Y. Chai, Macromolecules, 2017,

50, 4597-4609

110. H. Xiang, K. Shin, T. Kim, S. Moon, T.J. McCarthy, and T.P. Russell, J. Polym. Sci. B: Polym. Phys., 2005, 43, $3377-3383$

111. S. Jeon, Y. Lee, J. Kim, J. Lee, K. Char, and B. Sohn, React. Funct. Polym., 2009, 69, 558-563.

112. X. He, M. Song, H.J. Liang, and C.Y. Pan, J. Chem. Phys., 2001, 114, 10510-1-10510-9.

113. M. Y. Ha, J. H. Ryu, E. N. Cho, J. Choi, Y.J. Kim, and W. B. Lee, Phys. Rev. E, 2019, 100, 052502-1-0525027.

114. X. Liu, C. Zhou, H. Xi, Y. Zhou and W. Jiang, EPolymers, 2017, 17, 321-331.

115. G.J. Sevink, and A.V. Zvelindovsky, J. Chem. Phys., 2008, 128, 084901-1-084901-30.

116. J. Feng, H. Liu, and Y. Hu, Macromol. Theory Simul., 2006, 15, 674-85.

117. S. Li, X. Wang, L. Zhang, H. Liang, and P. Chen, Polymer, 2009, 50, 5149-5157.

118. Y. Zhu, and W. Jiang, Macromolecules, 2007, 40, $2872-2881$

119. M.-H. Cheng, H.-W. Ko, P.-Y. Chung, C.-W. Chang, J.-T. Chen, Soft Matter 12 (2016) 8087-8092.

120. C.-J. Chu, M.-H. Cheng, P.-Y. Chung, M.-H. Chi, K.S. Jeng, and J.-T. Chen. Int. J. Polym. Mater. Polym. Biomater., 2016, 65, 695-701.
121. C.-W. Chang, M.-H. Chi, H.-W. Ko, C.-W. Chu, Z.-X. Fang, Y.-H. Tu, and J.-T. Chen, Polym. Chem., 2017, 8, 3399-3404.

122. C.-W. Chang, Y.-H. Tu, K.-H. Luo, and J.-T. Chen, Langmuir, 2018, 34, 14388-14394.

123. Y.-L. Lin, M.-H. Cheng, C.-W. Chang, C.-W. Chu, C.T. Liu, and J.-T. Chen, Soft Matter, 2019, 15, 82018209.

124. N. Yan, Y. Sheng, H. Liu, Y. Zhu, and W. Jiang, Langmuir, 2015, 31, 1660-1669.

125. W. Zhao, D. Chen, Y. Hu, G.M. Grason, and T.P. Russell, ACS Nano, 2011, 5, 486-492.

126. M.-H. Cheng, Y.-C. Hsu, C.-W. Chang, H.-W. Ko, P.-

Y. Chung, and J.-T. Chen, ACS Appl. Mater. Interfaces, 2017, 9, 21010-21016.

127. Y. Kim, J. Mun, G. Yu, and K. Char, Macromol. Res., $2017,25,656-661$.

128. J. Xu, K. Wang, R. Liang, Y. Yang, H. Zhou, X. Xie, and J. Zhu, Langmuir, 2015, 31, 12354-12361.

129. X. Yue, Z. Geng, N. Yan, and W. Jiang, Phys. Chem. Chem. Phys., 2020, 22, 1194-1203.

130. C. Su, G. Shi, X. Li, X. Zhang, A.J. Muller, D. Wang, and G. Liu, Macromolecules, 2018, 51, 9484-9493.

131. C.-L. Liu, and H.-L. Chen, Soft Matter, 2018, 14, 5461-5468.

132. R.M. Michell, A.T. Lorenzo, A.J. Müller, M.C. Lin, H.L. Chen, I. Blaszczyk-Lezak, J. Martín, and C. Mijangos, Macromolecules, 2012, 45, 1517-1528. 
133. R.M. Michell, I. Blaszczyk-Lezak, C. Mijangos, A.J. Müller, Macromol. Symp., 2014, 337, 109-115.

134. G. Shi, G. Liu, C. Su, H. Chen, Y. Chen, Y. Su, A.J. Muller, and D. Wang, Macromolecules, 2017, 50, 9015-9023.

135. G. Shi, Y. Guan, G. Liu, A.J. Muller, and D. Wang, Macromolecules, 2019, 52, 6904-6912.

136. Y. Guan, G. Liu, G. Ding, T. Yang, A.J. Muller, and

D. Wang, Macromolecules, 2015, 48, 2526-2533.

137. H. Duran, M. Steinhart, H.-J. Butt, and G. Floudas,

Nano Lett., 2011, 11, 1671-1675.

138. J. Martin, A. Iturrospe, A. Cavallaro, A. Arbe, N. Stingelin, T.A. Ezquerra, C. Mijangos, and A. Nogales, Chem. Mater., 2017, 29, 3515-3525.

139. Y. Suzuki, H. Duran, M. Steinhart, H.-J. Butt, G. Floudas, Macromolecules, 2014, 47, 1793-1800.

140. M.Y.E Yau, I. Gunkel, B. Hartmann-Azanza, W. Akram, Y. Wang, T. Thurn-Albrecht, and M. Steinhart, Macromolecules, 2017, 50, 8637-8646.

141. J. Martin, M. Campoy-Quiles, A. Nogales, M. Garriga, M.I. Alonso, A.R. Goni, and M. MartinGonzalez, Soft Matter, 2014, 10, 3335-3346.

142. N. Shingne, M. Geuss, B. Hartmann-Azanza, M. Steinhart, and T. Thurn-Albrecht, Polymer, 2013, 54, 2737-2744.

143. R.A. Whiter, C. Boughey, M. Smith, and S. KarNarayan, Energy Technol., 2018, 6, 928-934.

144. V. Bhavanasi, D.Y. Kusuma, and P.S. Lee, Adv. Energy Mat. 2014, 4, 1400723-1-1400723-8.
145. D. Guo, F. Zeng, and B. Dkhil, J. Nanosci. Nanotechnol., 2014, 14, 2086-2100.

146. J.P. Cannon, S.D. Bearden, and S.A. Gold, Composites: Part A, 2010, 41, 836-841.

147. K. Shin, E. Woo, Y.G. Jeong, C. Kim, J. Huh, and K.W. Kim, Macromolecules, 2007, 40, 6617-6623.

148. H. Wu, W. Wang, Y. Huang, and Z. Su, Macromol. Rapid Commun., 2009, 30, 194-198.

149. C.-L. Liu, and H.-L. Chen, Macromolecules, 2017, 50, 631-641.

150. M. Avrami, J. Chem. Phys., 1941, 9, 177-184.

151. M.T. Casas, R.M. Michell, I. Blaszczyk-Lezak, J. Puiggali, C. Mijangos, A.T. Lorenzo, and A.J. Muller, Polymer, 2015, 70, 282-289.

152. B. Yu, P.C. Sun, T.H. Chen, Q.H. Jin, D.T. Ding, B.H. Li, and A.C. Shi, Phys. Rev. Lett., 2006, 96, 138306-1138306-4.

153. S.Z.D. Cheng, and A. Keller, Annu. Rev. Mater. Sci., 1998, 28, 533-562.

154. S. Ok, B. Hartmann, H. Duran, H. Eickmeier, M. Haase, U. Scheler, and M. Steinhart, J. Polym. Sci., Part B: Polym. Phys., 2019, 57, 1402-1408.

155. S. Ok, Magn. Reson. Chem., 2015, 53, 130-134.

156. M. Rubinstein, and R.H. Colby, Polymer Physics, Oxford University Press, New York, 2003.

157. K. Saalwächter, in NMR Methods for Characterization of Synthetic and Natural Polymers, ed. R. Zhang, T. Miyoshi, and P. Sun, Royal Society of Chemistry, Cambridge, 2019, Chapter 1, pp. 1-22. 
158. N. Fatkullin, T. Ikehara, H. Jinnai, S. Kawata, R. Kimmich, T. Nishi, Y. Nishikawa, and H.-B. Sun, NMR 3D Analysis Photopolymerization, Springer, New York, 2004.

159. S. Alexandris, G. Sakellariou, M. Steinhart, and G. Floudas, Macromolecules, 2014, 47, 3895-3900.

160. C. Politidis, S. Alexandris, G. Sakellariou, M. Steinhart, and G. Floudas, Macromolecules, 2019, 52, 4185-4195.

161. C.H. Tu , J. Zhou, M. Doi, H.-J. Butt, G. Floudas Phys. Rev. Let., 2020 ,125, 127802.

162. S. Ok, M. Steinhart, A. Serbescu, C. Franz, F.V. Chavez, and K. Saalwachter, Macromolecules 2010, 43, 4429-4434.

163. M. Hofmann, A. Herrmann, S. Ok, C. Franz, D. Kruk, K. Sallwachter, M. Steinhart, and E.A. Rossler, Macromolecules, 2011, 44, 4017-4021.

164. F. Lange, P. Judenstein, C. Franz, B. HartmannAzanza, S. Ok, M. Steinhart, and K. Saalwachter, ACS Macro Lett., 2015, 4, 561-565.

165. C. Franz, F. Lange, Y. Golitsyn, B. HartmannAzanza, M. Steinhart, M. Krutyeva, and K. Saalwachter, Macromolecules, 2016, 49, 244-256.

166. Y. Yao, Y.Suzuki, J. Seiwert, M. Steinhart, H. Frey, H.-J. Butt, G. Floudas, Macromolecules, 2017, 50, 8755-8764.

167. A. Talik, M. Tarnacka, I. Grudzka-Flak, P. Maksym, M. Geppert-Rybczynska, K. Wolnica, E. Kaminska, K.
Kaminski, M. Paluch, Macromolecules, 2018, 51, $4840-4852$.

168. M. Tarnacka, A. Talik, E. Kaminska, M. GeppertRybczynska, K. Kaminski, and M. Paluch, Macromolecules, 2019, 52, 3516-3529.

169. K. Adrjanowicz, R. Winkler, K. Chat, D.M. Duarte, W. tu, A.B. Unni, M. Paluch, and K.L. Ngai, Macromolecules, 2019, 52, 3763-3774.

170. M. Krutyeva, A. Wischnewski, M. Monkenbusch, L. Willner, J. Maiz, C. Mijangos, A. Arbe, J. Colmenero, A. Radulescu, O. Holderer, M. Ohl, and D. Richter, D. Phys. Rev. Lett., 2013, 110, 108303.

171. M. Krutyeva, S. Pasini, M. Monkenbusch, J. Allgaler, J. Malz, C. Mijangos, B. Hartmann-Azanza, M. Steinhart, N. Jalarvo, and D. Richter, J. Chem. Phys. 2017, 146, 203306.

172. S. Alexandris, P. Papadopoulos, G. Sakellariou, M. Steinhart, H.-J. Butt, and G. Floudas, Macromolecules, 2016, 49, 7400-7414.

173. W.K. Kipnusu, M.M. Elmahdy, E.U. Mapesa, J. Zhang, W. Bohlmann, D-M. Smilgies, C.M. Papadakis, and F. Kremer, ACS Appl. Mater. Interfaces, 2015, 7, 12328-12338.

174. J. Maiz, W. Zhao, Y. Gu, J. Lawrence, A. Arbe, A. Alegra, T. Emrick, J. Colmenero, T.P. Russell, and C. Mijangos, Polymer, 2014, 55, 4057-4066.

175. S. Li, Y. Jiang, and J.Z.Y. Chen, Soft Matter, 2013,

9, 4843-4854. 
176. X. Tang, W. Chen, and L. Li, Macromolecules,

2019, 52, 3575-3591.

177. L. Sangroniz, D. Cavallo, and A.J. Muller, Macromolecules, 2020, 53, 4581-4604. 